Network Working Group

Request for Comments: 3202

Category: Standards Track
R. Steinberger Paradyne Networks

O. Nicklass

RAD Data Communications Ltd.

January 2002

\title{
Definitions of Managed Objects \\ for Frame Relay Service Level Definitions
}

Status of this Memo

This document specifies an Internet standards track protocol for the Internet community, and requests discussion and suggestions for improvements. Please refer to the current edition of the "Internet Official Protocol Standards" (STD 1) for the standardization state and status of this protocol. Distribution of this memo is unlimited.

Copyright Notice

Copyright (C) The Internet Society (2002). All Rights Reserved.

Abstract

This memo defines an extension of the Management Information Base (MIB) for use with network management protocols in TCP/IP-based internets. In particular, it defines objects for managing the Frame Relay Service Level Definitions.

Table of Contents

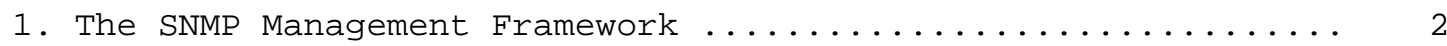

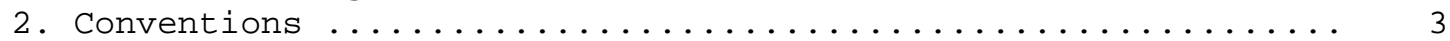

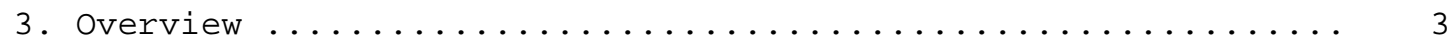

3.1. Frame Relay Service Level Definitions ................ 4

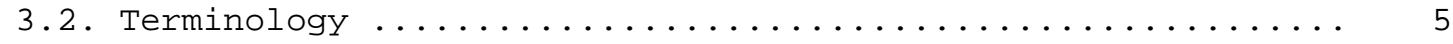

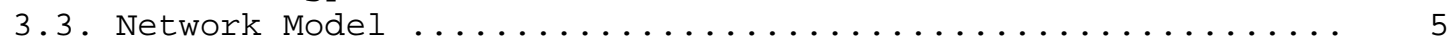

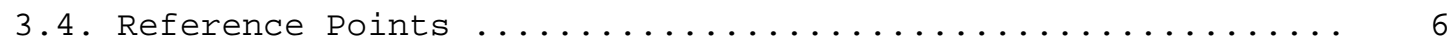

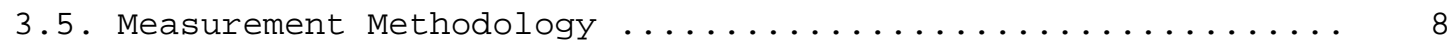

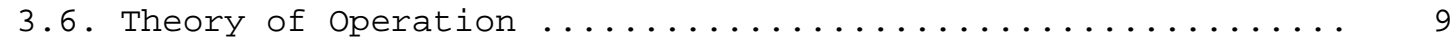

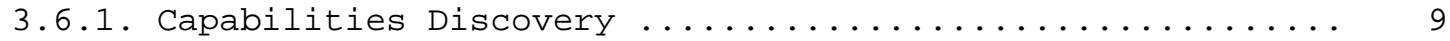

3.6.2. Determining Reference Points for Row Creation ......... 10

3.6.2.1. Graphical Examples of Reference Points ............. 11

3.6.2.1.1. Edge-to-Edge Interface Reference Point Example ...... 12

3.6.2.1.2. Edge-to-Edge Egress Queue Reference Point Example ... 13

3.6.2.1.3. End-to-End Using Reference Point Example ......... 14

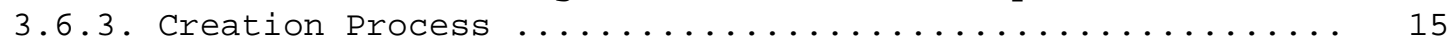

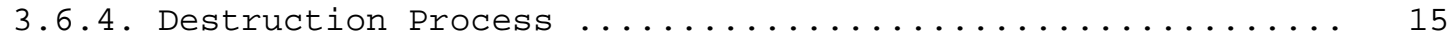




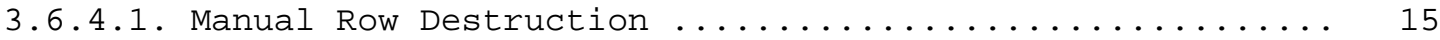

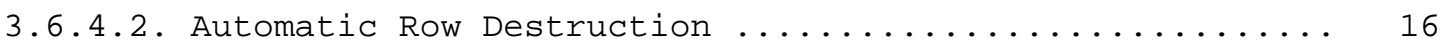

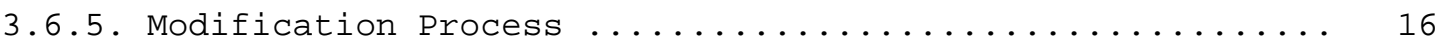

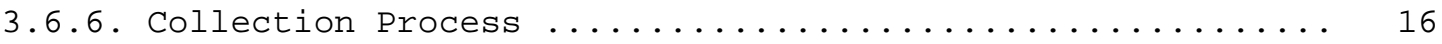

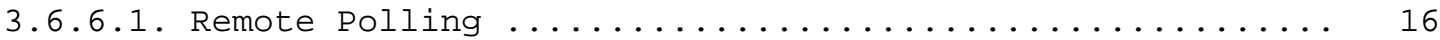

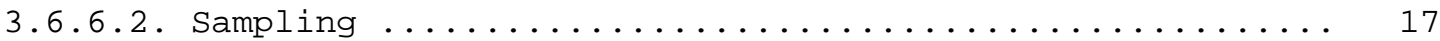

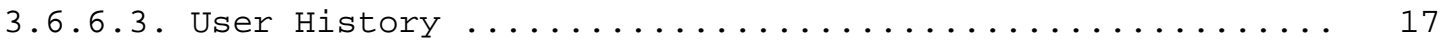

3.6.7. Use of MIB Module in Calculation of Service Level

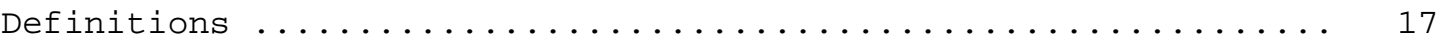

3.6 .8 . Delay .............................. 20

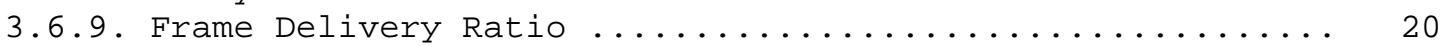

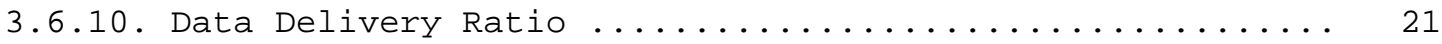

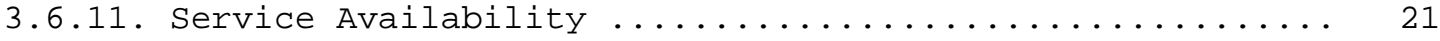

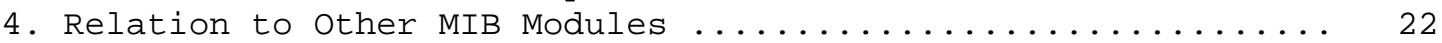

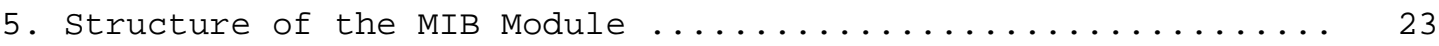

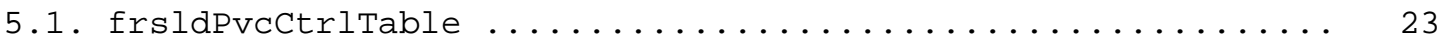

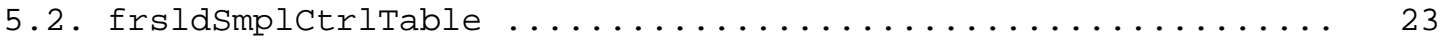

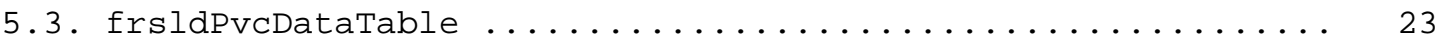

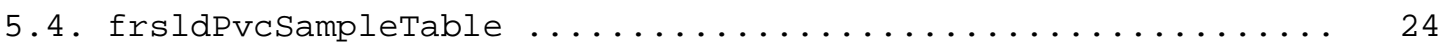

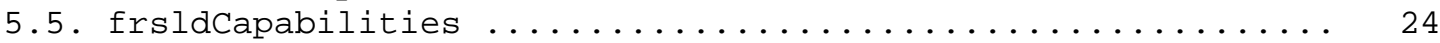

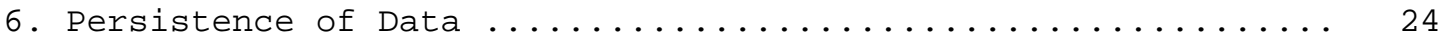

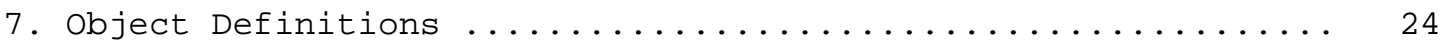

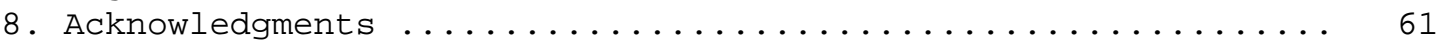

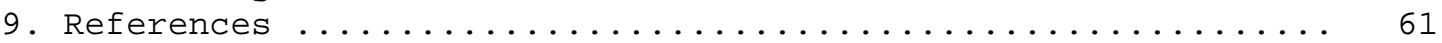

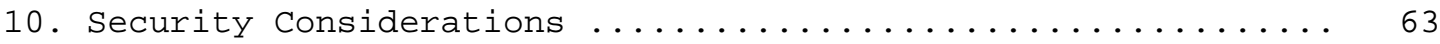

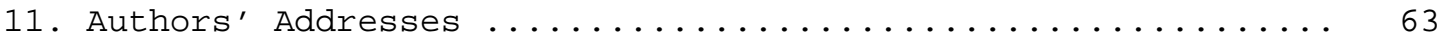

12. Full Copyright Statement ...................... 64

1. The SNMP Management Framework

The SNMP Management Framework presently consists of five major components:

- An overall architecture, described in RFC 2571 [1].

- Mechanisms for describing and naming objects and events for the purpose of management. The first version of this structure of Management Information (SMI) is called SMIv1 and described in STD 16, RFC 1155 [2], STD 16, RFC 1212 [3] and RFC 1215 [4]. The second version, called SMIv2, is described in STD 58, RFC 2578 [5], RFC 2579 [6] and RFC 2580 [7].

- Message protocols for transferring management information. The first version of the SNMP message protocol is called SNMPV1 and described in STD 15, RFC 1157 [8]. A second version of the SNMP message protocol, which is not an Internet standards track protocol, is called SNMPv2c and described in RFC 1901 [9] and RFC 
1906 [10]. The third version of the message protocol is called SNMPv3 and described in RFC 1906 [10], RFC 2572 [11] and RFC 2574 [12].

- Protocol operations for accessing management information. The first set of protocol operations and associated PDU formats is described in STD 15, RFC 1157 [8]. A second set of protocol operations and associated PDU formats is described in RFC 1905 [13].

- A set of fundamental applications described in RFC 2573 [14] and the view-based access control mechanism described in RFC 2575 [15].

A more detailed introduction to the current SNMP Management Framework can be found in RFC 2570 [16].

Managed objects are accessed via a virtual information store, termed the Management Information Base or MIB. Objects in the MIB are defined using the mechanisms defined in the SMI.

This memo specifies a MIB module that is compliant to the SMIv2. A MIB conforming to the SMIv1 can be produced through the appropriate translations. The resulting translated MIB must be semantically equivalent, except where objects or events are omitted because no translation is possible (use of Counter64). Some machine readable information in SMIV2 will be converted into textual descriptions in SMIv1 during the translation process. However, this loss of machine readable information is not considered to change the semantics of the MIB.

2. Conventions

The keywords MUST, MUST NOT, REQUIRED, SHALL, SHALL NOT, SHOULD, SHOULD NOT, RECOMMENDED, NOT RECOMMENDED, MAY, and OPTIONAL, when they appear in this document, are to be interpreted as described in RFC 2119 [22].

\section{Overview}

This MIB module addresses the items required to manage the Frame Relay Forum's Implementation Agreement for Service Level Definitions (FRF.13 [17]). At present, this applies to these values of the iftype variable in the Internet-standard MIB:
o frameRelay (32)
- frameRelayservice (44) 
This section provides an overview and background of how to use this MIB module.

\subsection{Frame Relay Service Level Definitions}

The frame relay service level definitions address specific characteristics of a frame relay service that can be used to facilitate the following tasks:

- Evaluation of frame relay service providers, offerings or products.

o Measurement of Quality of Service.

- Enforcement of Service Level Agreements.

- Planning or describing a frame relay network.

The following parameters are defined in FRF.13 [17] as a sufficient set of values to accomplish the tasks previously stated.

- Delay - The amount of time elapsed, in microseconds, from the time a frame exits the source to the time it reaches the destination. NOTE: FRF.13 [17] defines this value in terms of milliseconds.

- Frame Delivery Ratio - The ratio of the number of frames delivered to the destination versus the number of frames sent by the source. This ratio can be further divided by inspecting either only the frames within the CIR or only the frames in excess of the CIR.

- Data Delivery Ratio - The ratio of the amount of data delivered to the destination versus the amount of data sent by the source. This ratio can be further divided by inspecting either only the data within the CIR or only the data in excess of the CIR.

- Service Availability - The amount of time the frame relay service was not available. There are three types of availability statistics defined in FRF.13 [17]: Mean Time to Repair, Virtual Connection Availability, and Mean Time Between Service Outages. The later two require information about the scheduled outage time. It is assumed that scheduled outage time information will be maintained by the network management software, so it is not included in the MIB module.

Consult FRF.13 [17] for more details. 


\subsection{Terminology}

- CIR - The Committed Information Rate (CIR) is the subscriber data rate (expressed in bits/second) that the network commits to deliver under normal network conditions [18].

- DLCI - Data Link Connection Identifier [18].

- Logical Port - This term is used to model the Frame Relay "interface" on a device [18].

o NNI - Network to Network Interface [18].

- Permanent Virtual Connection (PVC) - A virtual connection that has its end-points and bearer capabilities defined at subscription time [18].

- Reference Point (RP) - The point of reference within the network model at which the calculations or data collection takes place.

o UNI - User to Network Interface [18].

\subsection{Network Model}

The basic model, as illustrated in figure 1 below, contains two frame relay DTE endpoints connected to a network cloud via a frame relay UNI interface. The network cloud can contain zero or more internal frame relay NNI connections that interconnect multiple networks. The calculations and data collection can be performed at any reference point within the network.

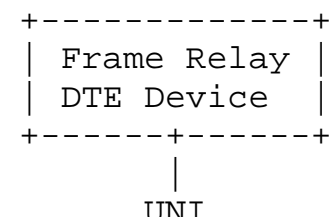

UNI

Connection

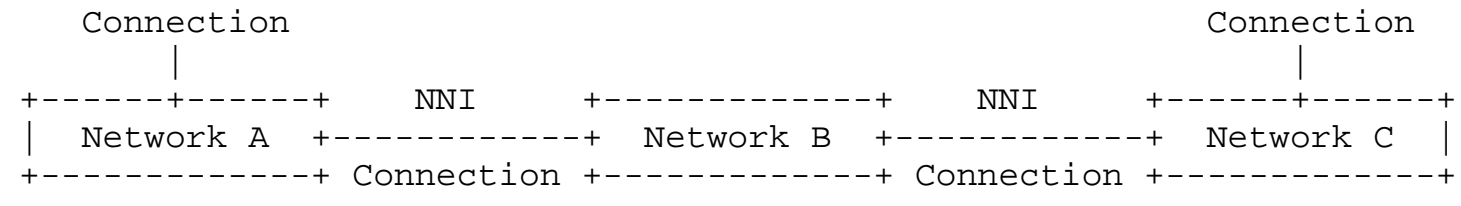

Figure 1

Frame Relay Network Reference Model 


\subsection{Reference Points}

The collection and calculations of the service level definitions apply to two reference points within the network. These two points are the locations where the frames are referenced in the collection of the service level specific information. The reference points used in the MIB module are shown in figure 2 below. For completeness, the module also allows for proprietary reference points which MAY exist anywhere in the network that is not a previously defined reference point. The meaning of the proprietary reference points is insignificant unless defined by the device manufacturer. 


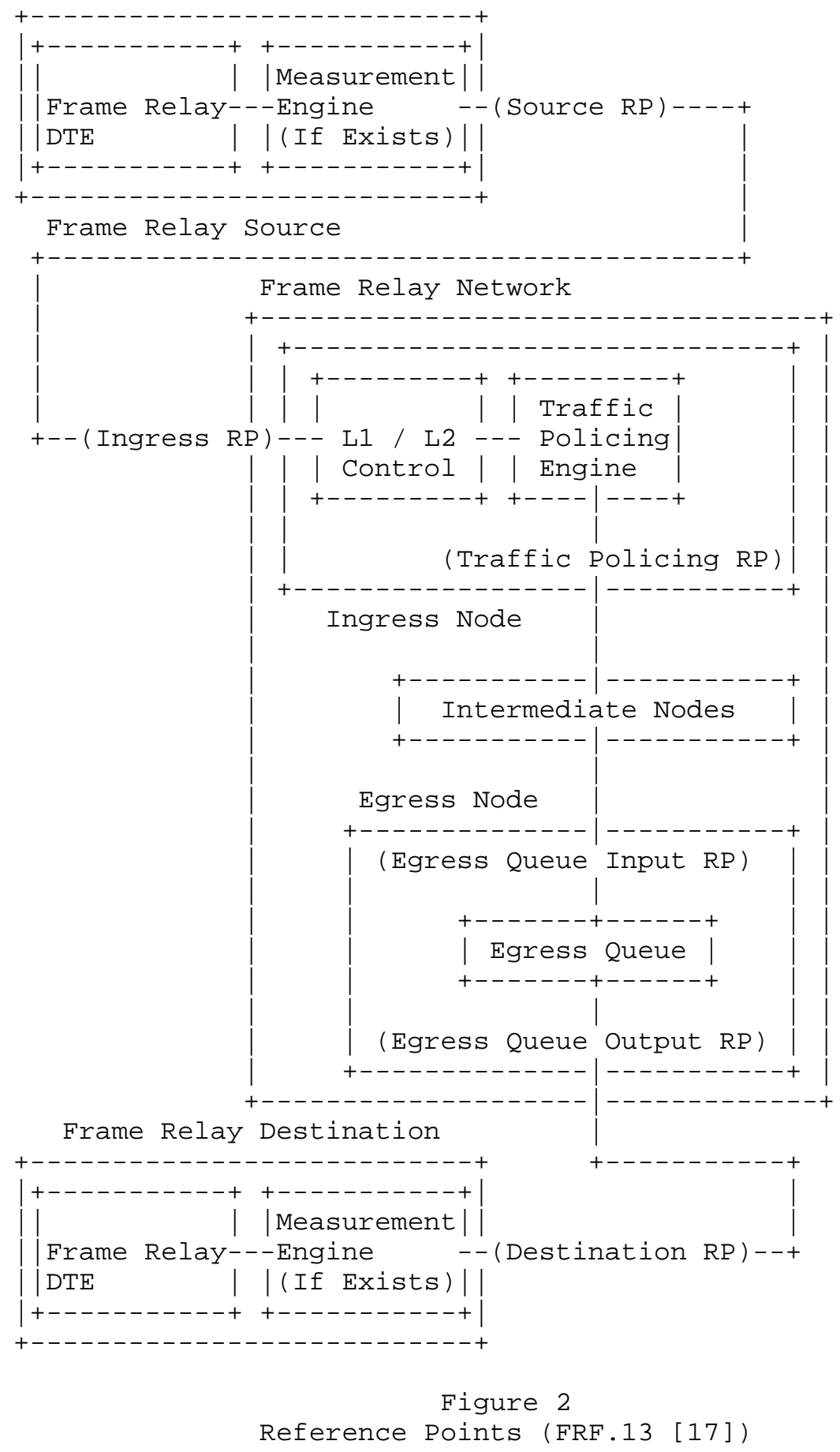


The MIB variables frsldPvcCtrlTransmitRP and frsldPvcCtrlReceiveRP allow the user to view and configure the reference points at which the calculations occur. These variables are specific to the device on which they are located. Frame relay devices act as both frame sources and frame destinations. The definitions in this MIB module apply to the interaction of a pair of devices on the network path. The same device can potentially use different reference points for calculation and collection of the statistics based on whether the referenced frame is sent or received by the device. When the device is acting as a frame source, the value of frsldPvcCtrlTransmitRP reflects the reference point used for all source calculations pertaining to the specified PVC. When the device is acting as a frame destination, the value of frsldPvcCtrlReceiveRP reflects the reference point used for all destination calculations pertaining to the specified PVC.

For example, FRF.13 [17] defines an Edge-to-Edge Egress Queue measurement domain as a domain in which measurement is performed between an Ingress Reference Point and an Egress Queue Input Reference Point. For this domain between a source device and a destination device, the value of frsldPvcCtrlTransmitRP for the source device would be set to ingTxLocalRP(2) and the value of frsldPvcCtrlReceiveRP for the destination device would be set to eqiRxLocalRP (4). While it is usually the case that the reference points would be equivalent on the remote device when monitoring frames going in the opposite direction, there is no requirement for them to be so.

It can be seen from the above example that a total of four reference points are required in order to collect information for both directions of traffic flow. The reference points represent the transmit and receive directions at both ends of a PVC. If a device has knowledge of the information from the remote device, it is possible to collect the statistics from a single device. This is not always the case. In most instances, two devices will need to be monitored to capture a complete description of the service level on a PVC. The reference points a single device is capable of monitoring are contained in the frsldRPCaps object.

\subsection{Measurement Methodology}

This document neither recommends nor suggests a method of implementation. This is left to the device manufacturer and should be independent of the data that is actually collected.

Periodic collection of this data can be performed through either polling of the data table, use of the sample tables or use of the user history group of RFC 2021 [19]. 


\subsection{Theory of Operation}

The following sections describe how to use this MIB module. They include row handling, data collection and data calculation. The recommendations here in are suggestions as to implementation and do not infer that they are the only method that can be used to perform such operations.

\subsubsection{Capabilities Discovery}

Three objects are provided specifically to aid the network manager in discovering the capabilities of the device with respect to this MIB module.

- frsldPvcCtrlWriteCaps This object reports the write capabilities of the PVC Control Table. Use this object to determine which objects can be modified. This need only be referenced if row creation or modification is to be performed.

- frsldSmplCtrlWriteCaps This object reports the write capabilities of the Sample Control Table. Use this object to determine which objects can be modified. The group need only be referenced if the sample tables will be used to collect historical information.

- frsldRPCaps

This object reports the reference points at which the device is capable of collecting information. This object needs to be referenced if row creation is to be performed in the PVC Control Table. Devices can only create rows containing supported reference points.

These objects do not imply that there is no need for an Agent Capabilities macro for devices that do not fully support every object in this MIB module. They are provided specifically to aid in the ensured network management operations of this MIB module with respect to row creation and modification.

An additional four objects are provided to report and control memory the utilization of this MIB module. These objects are frsldMaxPveCtrls, frsldNumPvcCtrls, frsldMaxsmplctrls are frsldNumSmplCtrls. Together, they allow a manager to control the 
amount of memory allocated for specific utilization by this MIB module. This is done by setting the maximum allowed allocation of controls.

\subsubsection{Determining Reference Points for Row Creation}

The performance of a PVC is monitored by evaluating the unidirectional flow of frames from an ingress point to an egress point. Reference points describe where each of the two measurements are made. Monitoring both of the uni-directional flows that make-up the PVC frame traffic requires a total of four reference points as shown in Figures 3 through 5. A monitoring point that evaluates traffic is restricted to counting frames that pass the reference points hosted locally on the monitoring point. Thus, if the monitoring point is near the ingress point of the flow, it will count the frames entering into the frame relay network. The complete picture of frame loss for the uni-directional flow requires information from the downstream reference point located at another (remote) monitoring point.

The local monitoring point MAY be implemented in such way that the information from the downstream monitoring point is moved to the local monitoring point using implementation-specific mechanisms. In this case all information required to calculate frame loss becomes available from the local measurement point. The local measurement point agent is capable of reporting all the objects in the FrsldPvcDataEntry row - the counts for offered frames entering the network and delivered frames exiting the network.

Alternatively, the local monitoring point MAY be restricted to counts of frames observed on the local device only. In this case, the objects of the FrsldPvcDataEntry row reporting what happened on the remote device are not available.

The following list shows the possible valid reference points for an FRF.13 SLA from the source reference point to the destination reference point in both directions.

- Local Information Only

Local Device: srcLocalRP, desLocalRP Remote Device: srcLocalRP, desLocalRP

- Remote Information Only

Local Device: srcRemoteRP, desRemoteRP Remote Device: srcRemoteRP, desRemoteRP 
- Mixed Two Device Model 1 (Local Device Always Transmitter)

Local Device: srcLocalRP, desRemoteRP

Remote Device: srcLocalRP, desRemoteRP

- Mixed Two Device Model 2 (Local Device Always Receiver)

Local Device: srcRemoteRP, desLocalRP

Remote Device: srcRemoteRP, desLocalRP

- Mixed One Device Model 1 (Directional Rows)

First Row: srcRemoteRP, desLocalRP (Receiver Row)

Second Row: srcLocalRP, desRemoteRP (Sender Row)

- Mixed One Device Model 2 (Device Based Rows)

First Row: srcLocalRP, desLocalRP (Local Row)

Second Row: srcRemoteRP, desRemoteRP (Remote Row)

Each of the above combinations is valid and provides the same information.

The following steps are recommended to find which reference points need to be configured:

1) Locate both of the devices at either end of the PVC to be monitored.

2) Determine the capabilities by referencing the frsldRPCaps object of each device.

3) Locate the best combination of the two devices such that the necessary reference points are all represented.

4) If any one of the necessary reference points does not exist in the combination of the two devices, it is not possible to monitor the FRF.13 defined SLA between the two reference point on the PVC.

3.6.2.1. Graphical Examples of Reference Points

FRF.13 [17] defines three specific combinations of reference points: Edge-to-Edge Interface, Edge-to-Edge Egress Queue and End-to-End.

Examples of valid reference points that may be used for each of these are discussed in the sections below. 
It is often the case that a device knows as a minimum either only local information or both local and remote information. Because these are two common examples, each will be illustrated below.

3.6.2.1.1. Edge-to-Edge Interface Reference Point Example

Device 1

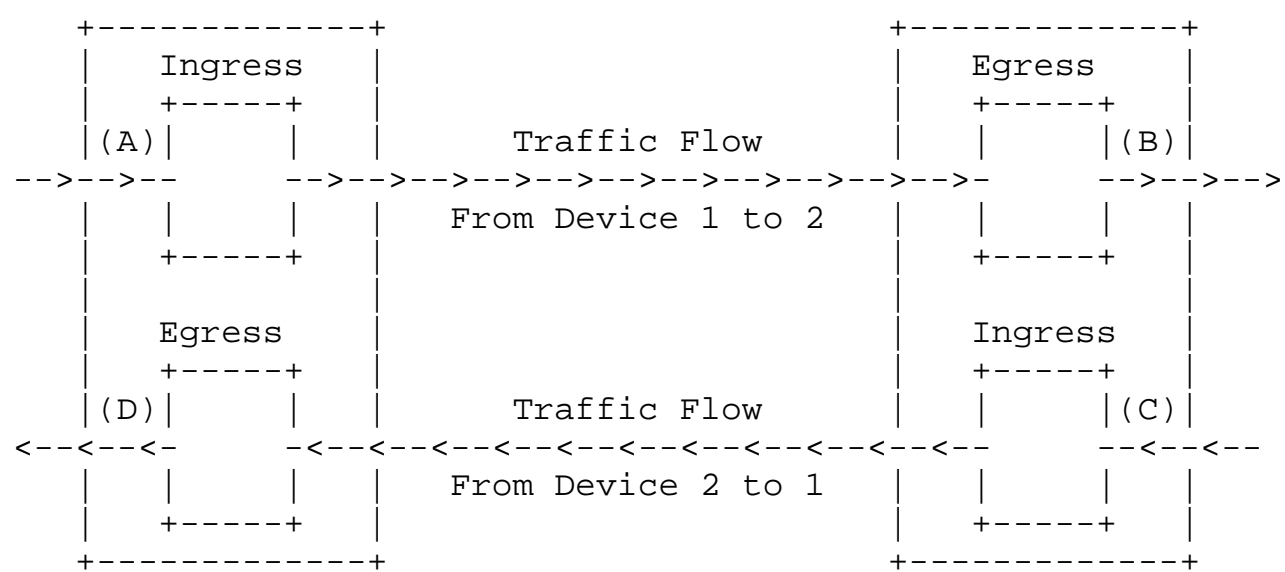

where (A), (B), (C) and (D) are reference points

For devices with only local knowledge, one row is required on each device as follows:

(A) frsldPvcCtrlTransmitRP for Device 1 = ingTxLocalRP (2)

(B) frsldPvcCtlrReceiveRP for Device 2 = eqoRxLocalRP (5)

(C) frsldPvcCtrlTransmitRP for Device 2 = ingTxLocalRP (2)

(D) frsldPvcCtlrReceiveRP for Device 1 = eqoRxLocalRP (5)

In which a single row is created on Device 1 containing reference points (A) and (D), and a single row is created on Device 2 containing reference points (C) and (B).

For devices with both local and remote knowledge, the two rows can exist in any combination on either device. For this example, the transmitting devices will be responsible for information regarding the flow for which they are the origin. Only one row is required per device for this example. 
(A) frsldPvcCtrlTransmitRP for Device 1 = ingTxLocalRP (2)

(B) frsldPvcCtlrReceiveRP for Device 1 = eqoRxRemoteRP (11)

(C) frsldPvcCtrlTransmitRP for Device 2 = ingTxLocalRP (2)

(D) frsldPvcCtlrReceiveRP for Device 2 = eqoRxRemoteRP(11)

3.6.2.1.2. Edge-to-Edge Egress Queue Reference Point Example

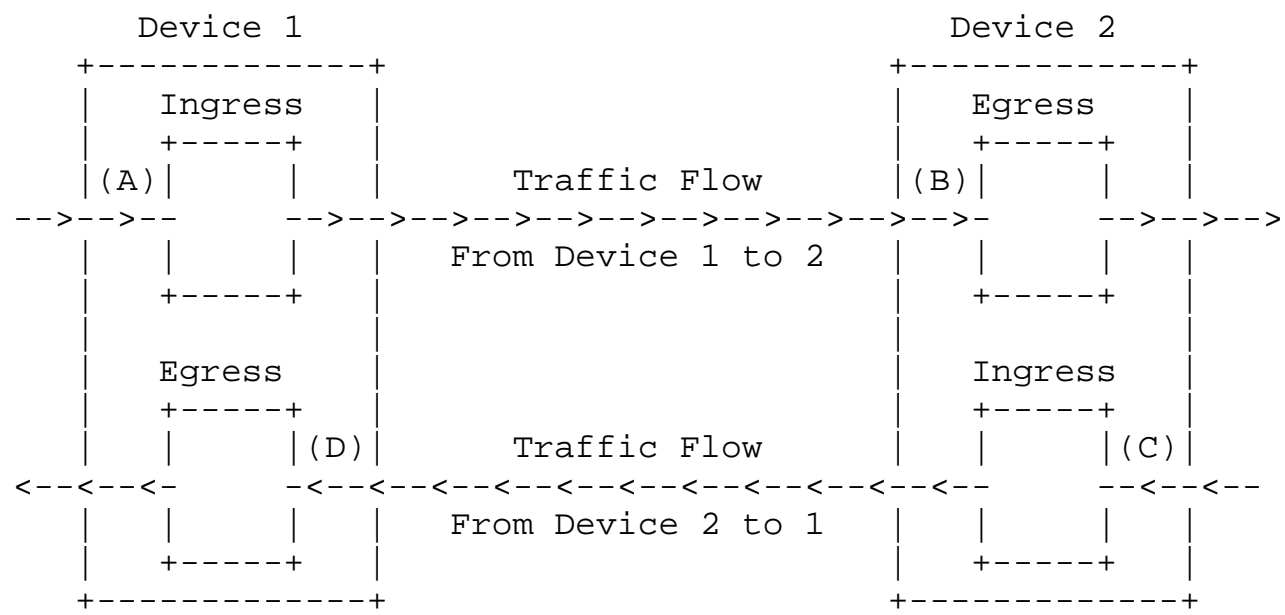

where (A), (B), (C) and (D) are reference points

Figure 4

For devices with only local knowledge, one row is required on each device as follows:

(A) frsldPvcCtrlTransmitRP for Device 1 = ingTxLocalRP (2)

(B) frsldPvcCtlrReceiveRP for Device 2 = eqiRxLocalRP (4)

(C) frsldPvcCtrlTransmitRP for Device 2 = ingTxLocalRP (2)

(D) frsldPvcCtlrReceiveRP for Device 1 = eqiRxLocalRP (4)

In which a single row is created on Device 1 containing reference points (A) and (D), and a single row is created on Device 2 containing reference points (C) and (B). 
For devices with both local and remote knowledge, the two rows can exist in any combination on either device. For this example, the transmitting devices will be responsible for information regarding the flow for which they are the origin. Only one row is required per device for this example.

(A) frsldPvcCtrltransmitRP for Device 1 = ingTxLocalRP (2)

(B) frsldPvcCtlrReceiveRP for Device 1 = eqiRxRemoteRP(10)

(C) frsldPvcCtrlTransmitRP for Device 2 = ingTxLocalRP(2)

(D) frsldPvcCtlrReceiveRP for Device 2 = eqiRxRemoteRP(10)

3.6.2.1.3. End-to-End Using Reference Point Example

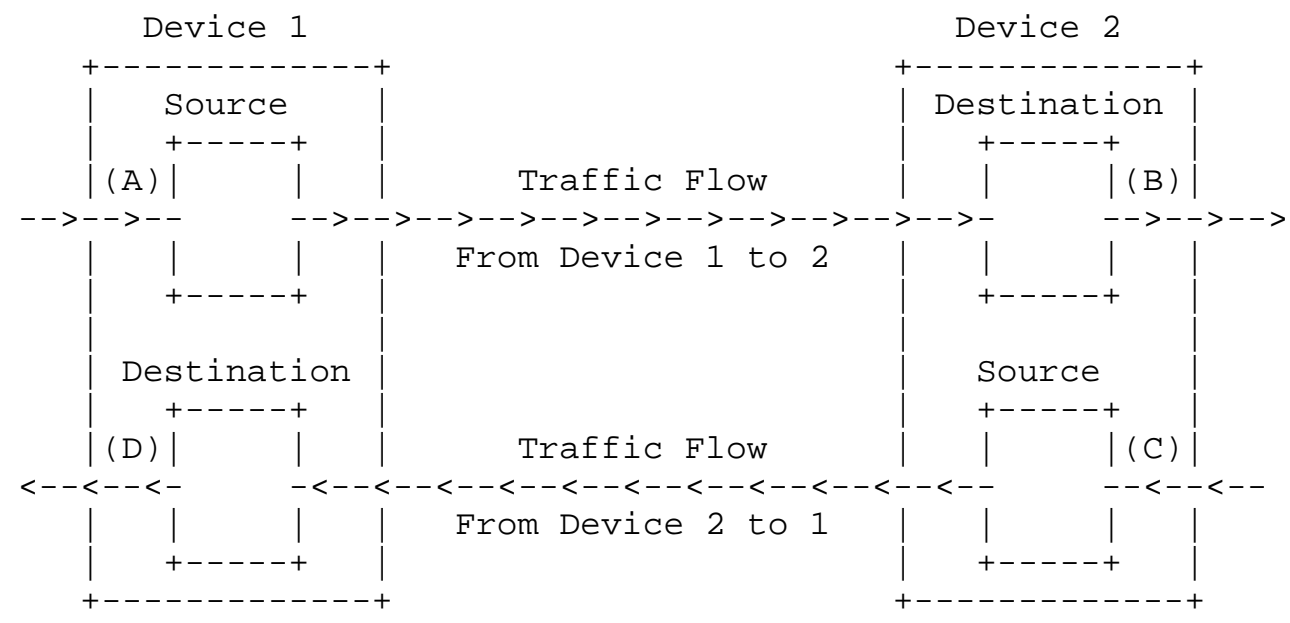

where (A), (B), (C) and (D) are reference points

Figure 5

For devices with only local knowledge, one row is required on each device as follows:
(A) frsldPvcCtrlTransmitRP for Device $1=\operatorname{srcLocalRP}(1)$
(B) frsldPvcCtlrReceiveRP for Device 2 = desLocalRP(1)
(C) frsldPvcCtrlTransmitRP for Device $2=\operatorname{srcLocalRP}(1)$
(D) frsldPvcCtlrReceiveRP for Device 1 = desLocalRP(1) 
In which a single row is created on Device 1 containing reference points (A) and (D), and a single row is created on Device 2 containing reference points (C) and (B).

For devices with both local and remote knowledge, the two rows can exist in any combination on either device. For this example, the transmitting devices will be responsible for information regarding the flow for which they are the origin. Only one row is required per device for this example.

(A) frsldPvcCtrlTransmitRP for Device $1=\operatorname{srcLocalRP}(1)$

(B) frsldPvcCtlrReceiveRP for Device 1 = desRemoteRP (7)

(C) frsldPvcCtrlTransmitRP for Device $2=\operatorname{srcLocalRP}(1)$

(D) frsldPvcCtlrReceiveRP for Device 2 = desRemoteRP (7)

\subsubsection{Creation Process}

In some cases, devices will automatically populate the rows of PVC Control Table and potentially the Sample Control Table. However, in many cases, it may be necessary for a network manager to manually create rows.

Manual creation of rows requires the following steps:

1) Ensure the PVC exists between the two devices.

2) Determine the necessary reference points for row creation.

3) Create the row(s) in each device as needed.

4) Create the row(s) in the sample control tables if desired.

3.6.4. Destruction Process

\subsubsection{Manual Row Destruction}

Manual row destruction is straight forward. Any row can be destroyed and the resources allocated to it are freed by setting the value of its status object (either frsldPvcCtrlstatus or frsldSmplctrlstatus) to destroy (6). It should be noted that when frsldPvectrlstatus is set to destroy(6) all associated sample control, sample and data table rows will also be destroyed. Similarly, when frsldSmplCtrlstatus is set to destroy(6) all sample rows will also be 
destroyed. The frsldPvcCtrlPurge objects do not apply to manual row destruction. If the row is set to destroy(6) manually, the rows are destroyed as part of the set.

\subsubsection{Automatic Row Destruction}

Rows is the tables may be destroyed automatically based on the existence of the DLCI on which they rely. This behavior is controlled by the frsldPvcCtrlPurge and frsldPvcCtrlDeleteonPurge objects. When a DLCI no longer exists in the device, the data in the tables has no relation to anything known on the network. However, there may be some need to keep the historic information active for a short period after the destruction or removal of a DLCI. If the basis for the row no longer exists, the row will be destroyed at the end of the purge interval that is controlled by frsldPvcCtrlpurge.

The effects of automatic row destruction are the same as manual row destruction.

\subsubsection{Modification Process}

All read-create items in this MIB module can be modified at any time if they are fully supported. Write access is not required. To simplify the use of the MIB frsldPvcCtrlWriteCaps and frsldSmplCtrlWriteCaps state which of the read-create variables can actually be written on a particular device.

\subsubsection{Collection Process}

\subsubsection{Remote Polling}

This MIB module supports data collection through remote polling of the free running counters in the PVC Data Table. Remote polling is a common method used to capture real-time statistics. A remote management station polls the device to collect the desired information. It is recommended all statistics for a single PVC be collected in a single PDU.

The following objects are designed around the concept of real-time polling:

- frsldPvcDataMissedPolls

- frsldPvcDataFrDeliveredC

- frsldPvcDataFrDeliveredE

- frsldPvcDataFrofferedC

- frsldPvcDataFrofferedE

- frsldPvcDataDataDeliveredC

- frsldPvcDataDataDeliveredE 


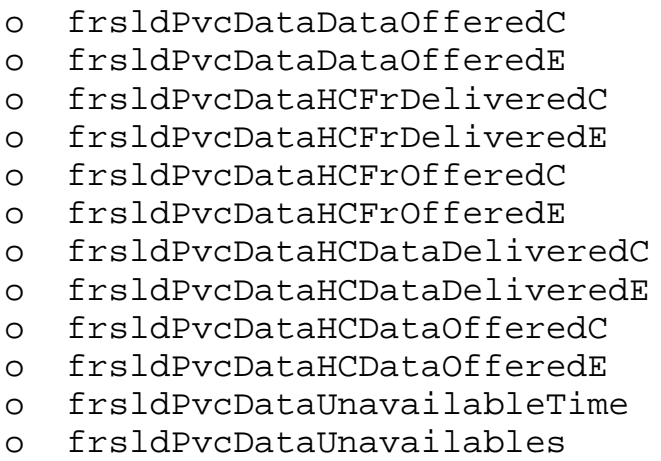

3.6.6.2. Sampling

The sample tables provide the ability to historically sample data without requiring the additional overhead of polling. At key periods, a network management station can collect the samples needed. This method allows the manager to perform the collection of data at times that will least affect the active network traffic.

The sample data can be collected using a series of SNMP getNext or getBulk operations. The value of frsldPvcSmplidx increments with each new collection bucket. This allows the managers to skip information that has already been collected. However, care should be taken in that the value can roll over after a long period of time.

The start and end times of a collection period allow the manager to know what the actual period of collection was. It is possible for there to be discontinuities in the sample table, so both start and end should be referenced.

\subsubsection{User History}

User history, as defined in RFC 2021 [19], is an alternative mechanism that can be used to get the same benefits as the sample table by using the objects provided for real-time polling. Some devices MAY have the ability to use user history and opt not to support the sample tables. If this is the case, the information from the data table can be used to define a group of user history objects.

3.6.7. Use of MIB Module in Calculation of Service Level Definitions

The objects in this MIB module can be used to calculate the statistics defined in FRF.13 [17]. The description below describes the calculations for one direction of the data flow, i.e., data sent from local transmitter to a remote receiver. A complete set of bidirectional information would require calculations based on both 
directions. For the purposes of this description, the reference points used SHOULD consistently represent data that is sent by one device and received by the other.

A complete evaluation requires the combination of two uni-directional flows. It is possible for a management station to combine all of the calculated information into one conceptual row. Doing this requires that each of the metrics are collected for both flow directions and grouped by direction If the information is split between two devices, the management station must know which two devices to communicate with for the collection of all information. The grouping of information SHOULD be from ingress to egress in each flow direction.

The calculations below use the following terminology:

- DelayAvg

The average delay on the PVC. This is represented within the MIB module by frsldPvcSmplDelayAvg.

- FrDeliveredC

The number of frames received by the receiving device through the receive reference point that were delivered within CIR. This is represented within the MIB module by one of frsldPvcDataFrDeliveredC, frsldPvcDataHCFrDeliveredC, frsldPvCSmplFrDeliveredC, or frsldPvCSmplHCFrDeliveredC.

o FrDeliveredE

The number of frames received by the receiving device through the receive reference point that were delivered in excess of CIR. This is represented within the MIB module by one of frsldPvcDataFrDeliveredE, frsldPvcDataHCFrDeliveredE, frsldPvcSmplFrDeliveredE, or frsldPvCSmplHCFrDeliveredE.

- FrofferedC

The number of frames offered by the transmitting device through the transmit reference point that were sent within CIR. This is represented within the MIB module by one of frsldPvcDataFrofferedC, frsldPvcDataHCFrOfferedC, frsldPvcSmplFrOfferedC, or frsldPvcSmplHCFrOfferedC. 
- FrofferedE

The number of frames offered by the transmitting device through the transmit reference point that were sent in excess of CIR. This is represented within the MIB module by one of frsldPvcDataFrofferedE, frsldPvcDataHCFrOfferedE, frsldPvcSmplFrofferedE, or frsldPvCSmplHCFrOfferedE.

- DataDeliveredC

The number of octets received by the receiving device through the receive reference point that were delivered within CIR. This is represented within the MIB module by one of frsldPvcDataDataDeliveredC, frsldPvcDataHCDataDeliveredC, frsldPvcSmplDataDeliveredC, or frsldPvcSmplHCDataDeliveredC.

- DataDeliveredE

The number of octets received by the receiving device through the receive reference point that were delivered in excess of CIR. This is represented within the MIB module by one of frsldPvcDataDataDeliveredE, frsldPvcDataHCDataDeliveredE, frsldPvcSmplDataDeliveredE, or frsldPvcSmplHCDataDeliveredE.

- DataOfferedC

The number of octets offered by the transmitting device through the transmit reference point that were sent within CIR. This is represented within the MIB module by one of frsldPvcDataDataOfferedC, frsldPvcDataHCDataofferedC, frsldPvcSmplDataOfferedC, or frsldPvcSmplHCDataOfferedC.

o DataOfferedE

The number of octets offered by the transmitting device through the transmit reference point that were sent in excess of CIR. This is represented within the MIB module by one of frsldPvcDataDataofferedE, frsldPvcDataHCDataOfferedE, frsldPvcSmplDataOfferedE, or frsldPvcSmplHCDataOfferedE.

o UnavailableTime

The amount of time the PVC was not available during the interval of interest. This is represented within the MIB module by either frsldPvcDataUnavailableTime or frsldPvcSmplUnavailableTime. 
o Unavailables

The number of times the PVC was declared to be unavailable during the interval of interest. This is represented within the MIB module by either frsldPvcDataUnavailables or frsldPvcSmplUnavailables.

3.6.8. Delay

The frame transfer delay is defined as the amount of time elapsed, in microseconds, from the time a frame exits the source to the time it reaches the destination. The average delay can be found using the MIB variable described in DelayAvg above. The delay may be calculated as either round trip or one way, and this information is held in the frsldPvcCtrlDelayType MIB variable. If the delay be calculated as round trip, the value of DelayAvg represents the average of the total delays of the round trips. In this case, the manager SHOULD divide the value returned by the agent by two to obtain the frame transfer delay. In the case that frsldPvcCtrlDelayType is oneWay, the value of DelayAvg represents the average of the frame transfer delays and SHOULD be used as is.

\subsubsection{Frame Delivery Ratio}

The frame delivery ratio is defined as the total number of frames delivered to the destination divided by the frames offered by the source. The destination values can be obtained using FrDeliveredC and FrDeliveredE. The source values can be obtained using FrofferedC and FrofferedE.

$\begin{aligned} & \text { Frame Delivery Ratio }=\begin{array}{r}\text { FrDeliveredC }+ \text { FrDeliveredE } \\ - \\ \text { FrOfferedC }+ \text { FrofferedE }\end{array} \begin{array}{c}\text { FrDeliveredC } \\ ----------\end{array} \\ & \text { FrofferedC } \\ & \text { Excess Frame Delivery Ratio }=\begin{array}{c}\text { FrDeliveredE } \\ ---------- \\ \text { FrofferedE }\end{array}\end{aligned}$




\subsubsection{Data Delivery Ratio}

The data delivery ratio is defined as the total amount of data delivered to the destination divided by the data offered by the source. The destination values can be obtained using DataDeliveredC and DataDeliveredE. The source values can be obtained using DataofferedC and DataOfferedE.

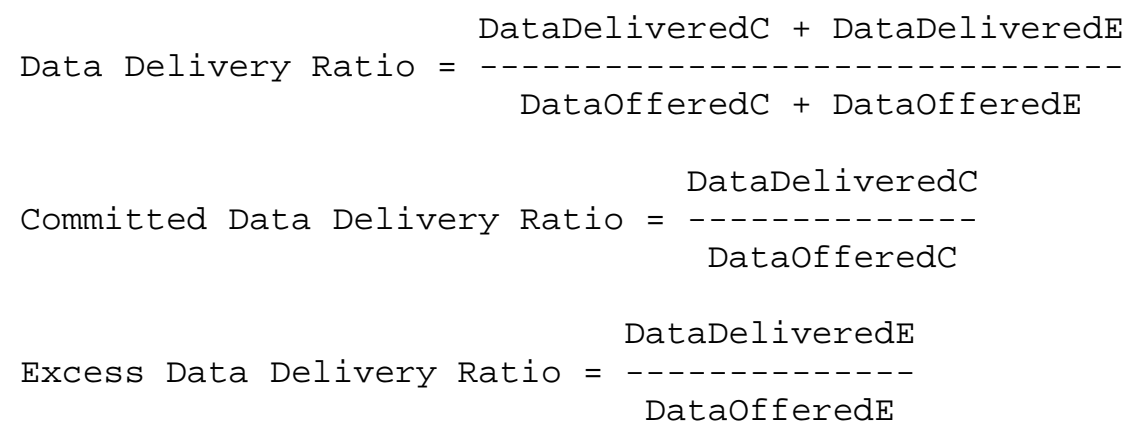

\subsubsection{Service Availability}

Some forms of service availability measurement defined in FRF.13 [17] require knowledge of the amount of time the network is allowed to be unavailable during the period of measurement. This is called the excluded outage time and will be represented in the measurements below as ExcludedTime. It is assumed that the management software will maintain this information in that it often relates to specific times and dates that many devices are not capable of maintaining. Further, it may change based on a moving maintenance window that the device cannot track well.

Mean Time to Repair (FRMTTR) $=0$ if Unavailables is 0.

UnavailableTime

Otherwise, FRMTTR = ---------------

Unavailables

Virtual Connection Availability (FRVCA) = 0 if IntervalTime equals ExcludedTime.

IntervalTime - ExcludedTime - UnavailableTime
Otherwise, FRVCA $=--{ }^{-} 00$
IntervalTime - ExcludedTime

Mean Time Between Service Outages (FRMTBSO) = 0 if Unavailables is 0. 
Otherwise, FRMTBSO = IntervalTime - ExcludedTime - UnavailableTime

Unavailables

4. Relation to Other MIB Modules

There is no explicit relation to any other frame relay MIB module nor are any required to implement this MIB module. However, there is a need for knowledge of ifIndexes and some understanding of DLCIs. The ifIndex information can be found in the IF-MIB [21] which is required. The DLCI information can be found in either the Frame Relay DTE MIB (RFC 2115) [20] or the Frame Relay Network Services MIB (RFC 2954) [18]; however, neither is required.

Upon setting of frsldPvcCtrlstatus in the frsldPvcCtrlTable to active(1) the system can be in one of the following three states:

(1) The respective DLCI is known and is active. This corresponds to a state in which frPVCEndptRowstatus is active(1) and frPVCEndptRcvdSigStatus is either active(2) or none(4) for the Frame Relay Network Services MIB (RFC 2954) [18]. For the Frame Relay DTE MIB, the same state is shown by frCircuitRowstatus of active(1) and frCircuitstate of active(2).

(2) The respective DLCI has not been created. This corresponds to a state in which the row with either frPVCEndptDLCIIndex or frCircuitDlci equal to the respective DLCI does not exist in either the frPVCEndptTable or the frCircuitTable respectively.

(3) The respective DLCI has just been removed. This corresponds to a state in which either frPVCEndptRowstatus is no longer active(1) or frPVCEndptRcvdSigStatus is no longer active(2) or none(4) for the Frame Relay Network Services MIB (RFC 2954) [18]. For the Frame Relay DTE MIB, the same state is shown when either frCircuitRowstatus is no longer active(1) or frCircuitstate is no longer active(2).

For the first case, the row in the frsldPvcDataTable will be filled. If frsldSmplCtrlStatus in the frsldSmplctrlTable for the respective DLCI is also 'active' the frsldPvcSampleTable will be filled as well.

For the second case, the respective rows will not be added to any of the data or sample tables and frsldPveCtrlstatus sHould report notReady (3). 
For the third case, frsldPvcCtrlDeleteonPurge should direct the behavior of the system. If all tables are purged, this case will be equivalent to the second case above. Otherwise, frsldPvcCtrlstatus SHOULD remain active(1).

5. Structure of the MIB Module

The FRSLD-MIB consists of the following components:

- frsldPvcCtrltable

- frsldSmplctrlTable

- frsldPvcDataTable

- frsldPvcSampletable

- frsldCapabilities

Refer to the compliance statement defined within for a definition of what objects MUST be implemented.

\section{1. frsldPveCtrltable}

The frsldPvcCtrlTable is the central control table for operations of the Frame Relay Service Level Definitions MIB. It provides variables to control the parameters required to calculate the objects in the other tables.

A row in this table MUST exist in order for a row to exist in any other table in this MIB module.

\section{2. frsldSmplCtrlTable}

This is an optional table to allow control of sampling of the data in the data table.

\section{3. frsldPveDataTable}

This table contains the calculated data. It relies on configuration from the control table. 


\section{4. frsldPvcSampleTable}

This table contains samples of the delivery and availability information from the data table as well as delay information calculated over the sample period. It relies on configuration from both the control table and the sample control table.

\section{5. frsldCapabilities}

This is a group of objects that define write capabilities of the read-create objects in the tables above.

6. Persistence of Data

The data in frsldPvcCtrlTable and frsldSmplCtrlTable SHOULD persist through power cycles. Note, however, that the symantics of readiness for the rows still applies. This means that it is possible for a row to be reprovisioned as notReady(3) if the underlying DLCI does not persist. The data collected in the other tables SHOULD NOT persist through power cycles in that the reference Timestamp is no longer valid.

7. Object Definitions

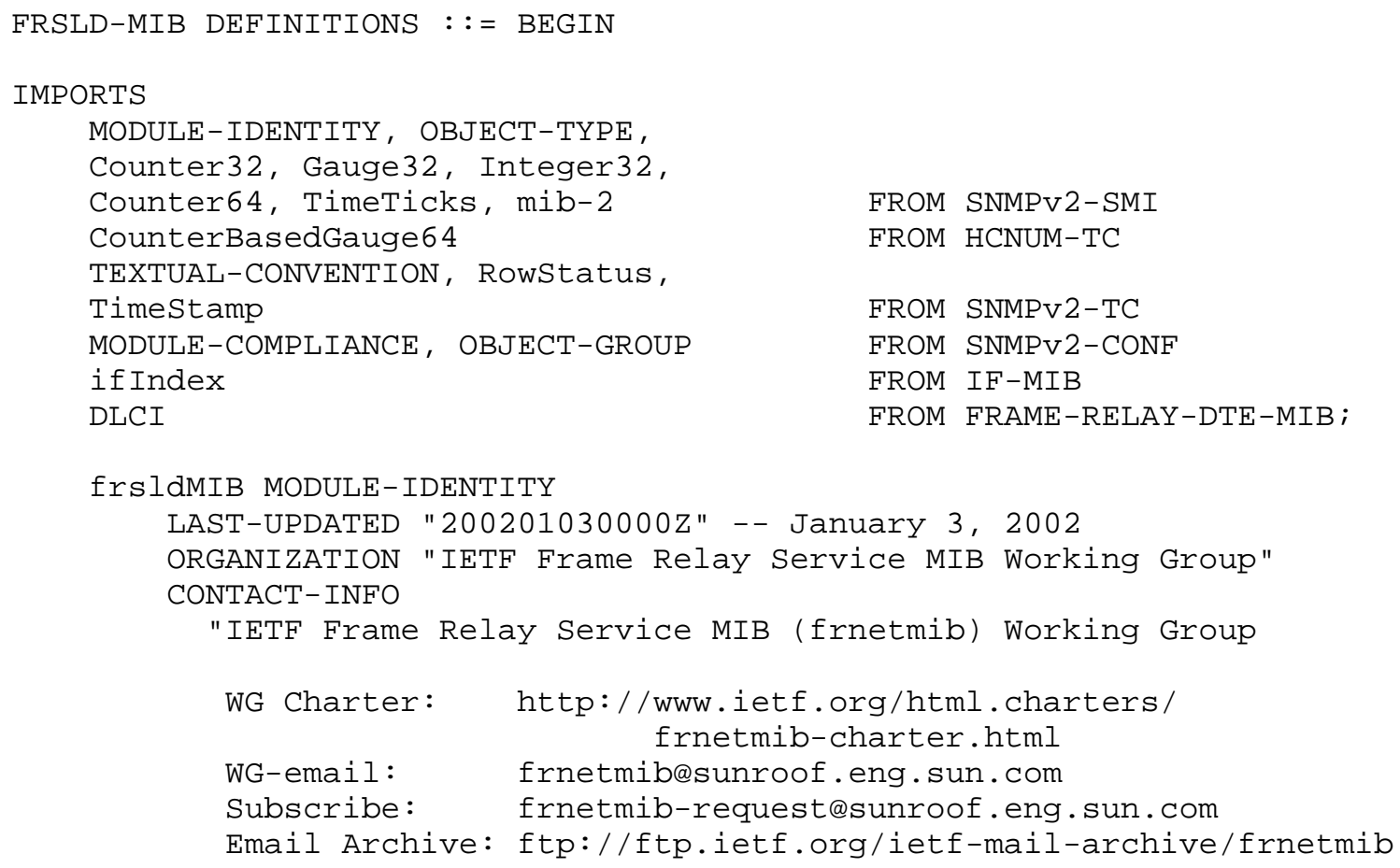




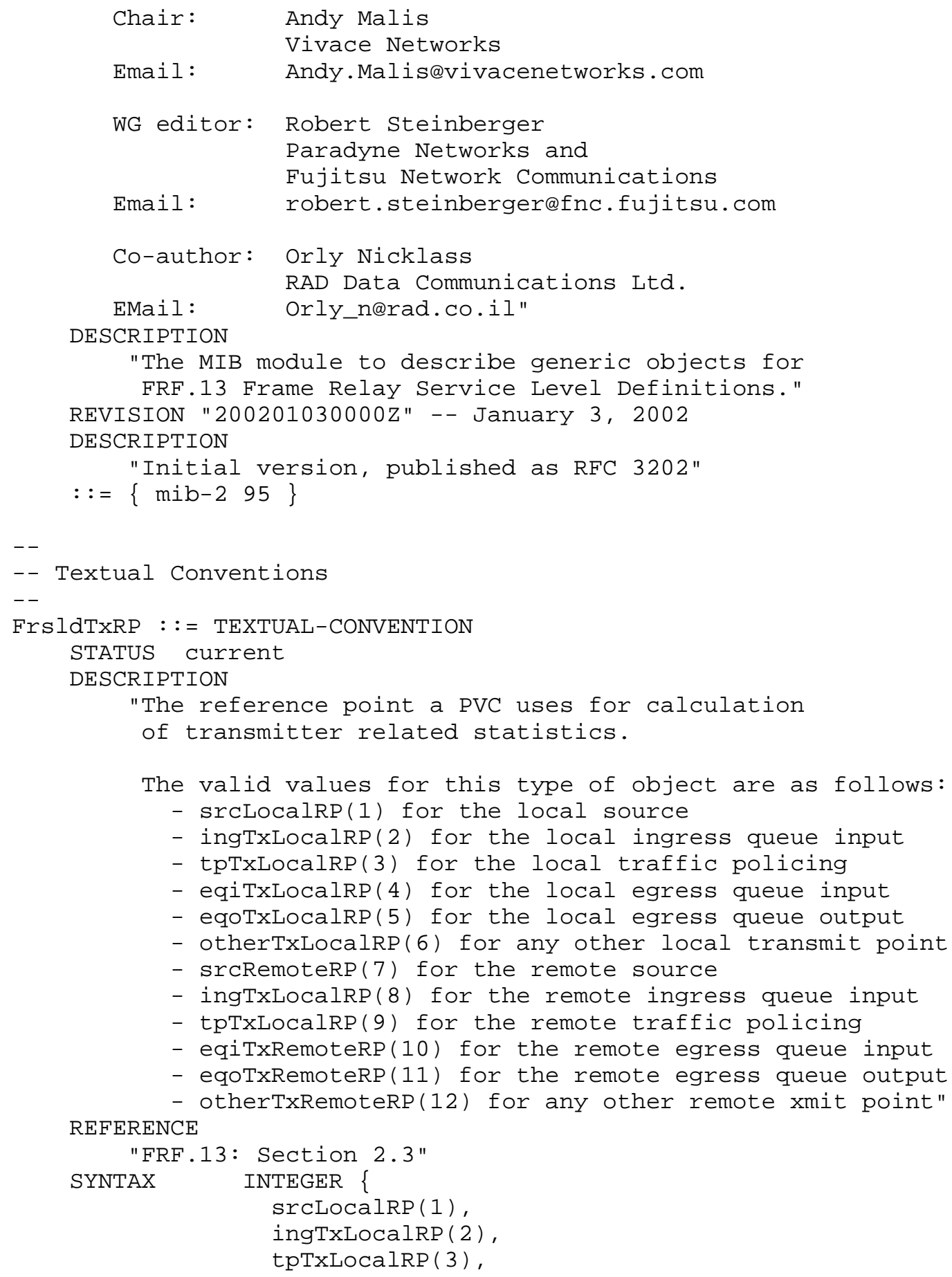




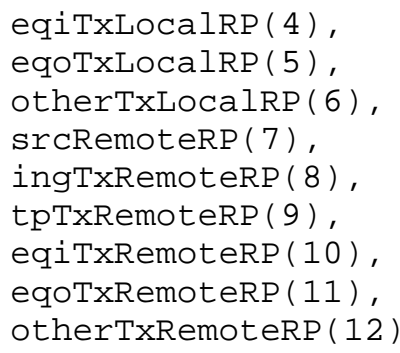


-- Base Objects

$---$

frsldObjects OBJECT IDENTIFIER : := frsldMIB 1$\}$

frsldCapabilities OBJECT IDENTIFIER ::= f frsldMIB 2$\}$

frsldConformance OBJECT IDENTIFIER ::= frsldMIB 3$\}$

-- The Frame Relay Service Level Definitions PVC Control Table

$--$

-- This table is used to define and display the parameters of

-- service level definitions on individual PVCs.

frsldPvcCtrlTable OBJECT-TYPE

SYNTAX SEQUENCE OF FrsldPvCCtrlEntry

MAX-ACCESS not-accessible

STATUS current

DESCRIPTION

"The Frame Relay Service Level Definitions

PVC control table."

$::=\{$ frsldobjects 1$\}$

frsldPvCCtrlEntry OBJECT-TYPE

SYNTAX FrsldPvCCtrlentry

MAX-ACCESS not-accessible

STATUS current

DESCRIPTION

"An entry in the Frame Relay Service Level

Definitions PVC control table."

INDEX \{ifIndex, frsldPvcCtrlDlci, frsldPvcCtrlTransmitRP, frsldPvcCtrlReceiveRP

$::=\{$ frsldPvcCtrlTable 1$\}$

FrsldPvCCtrlentry : := SEQUENCE \{

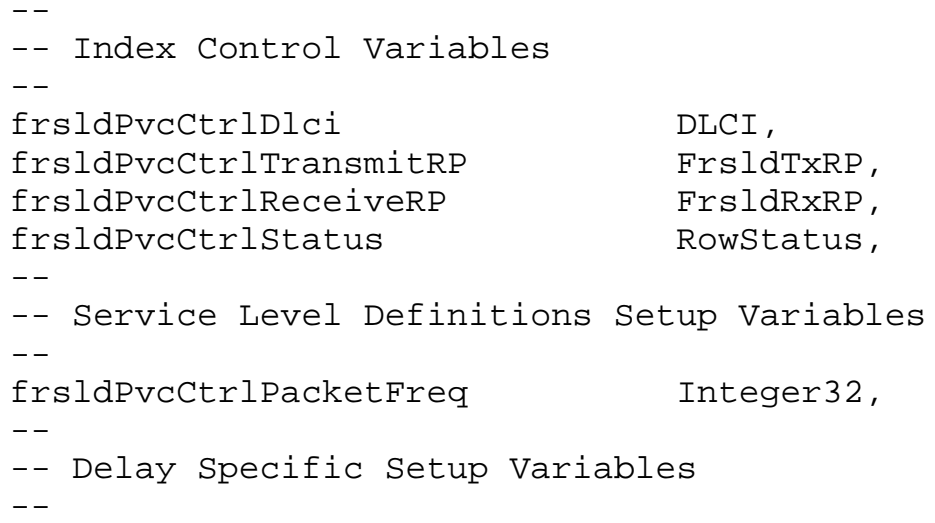




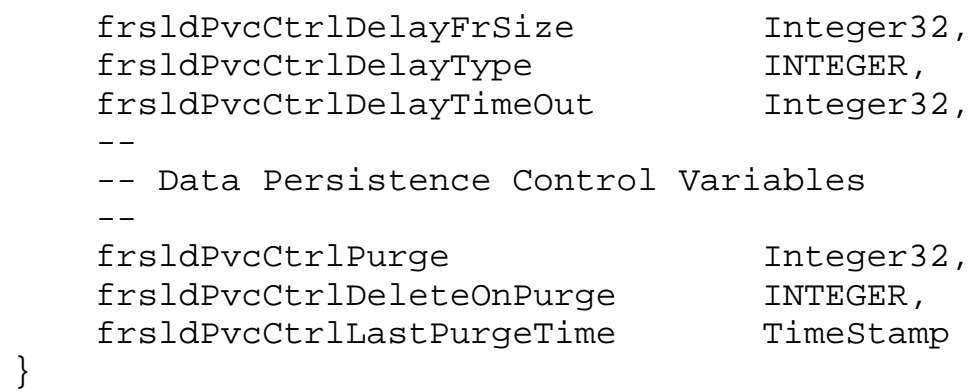




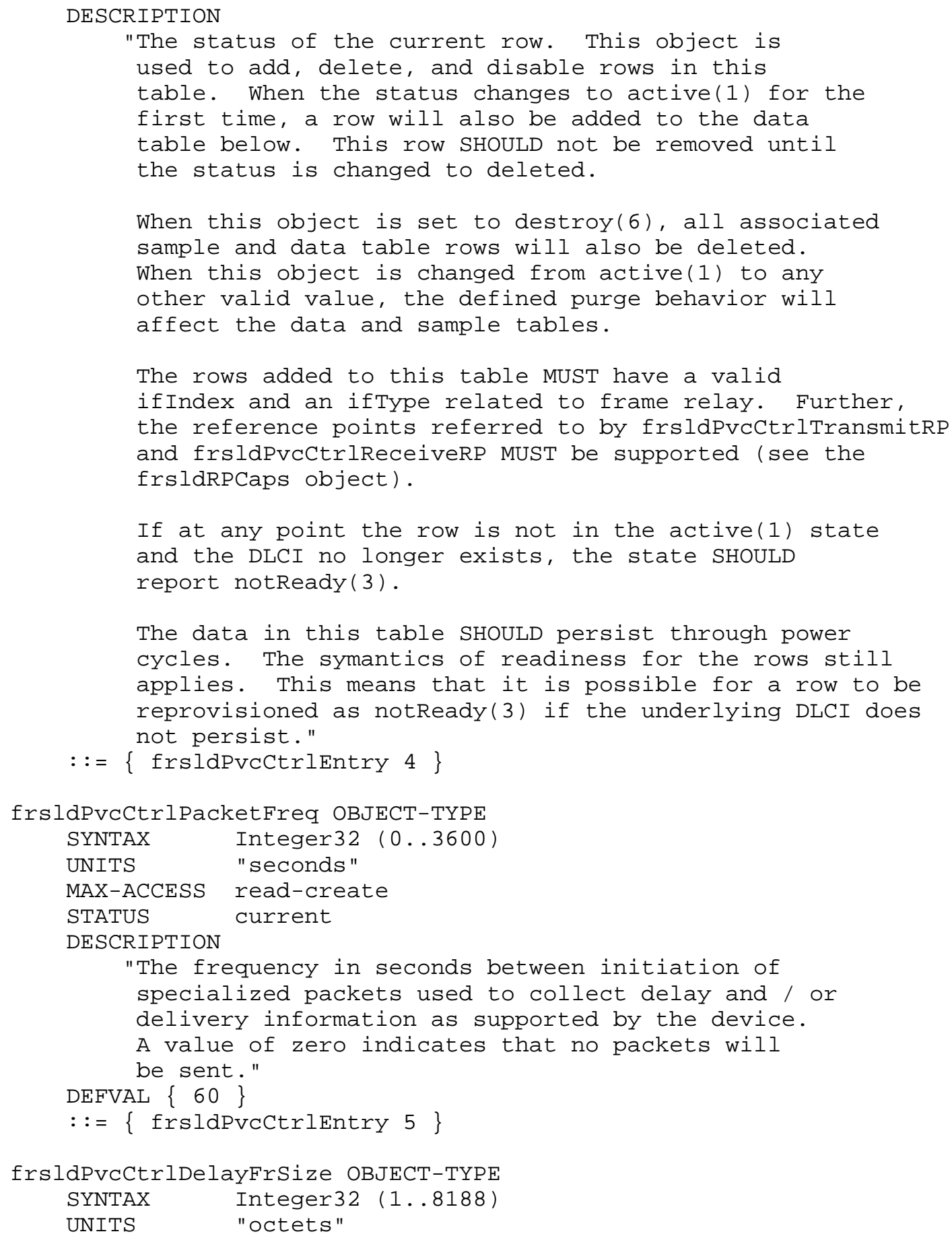




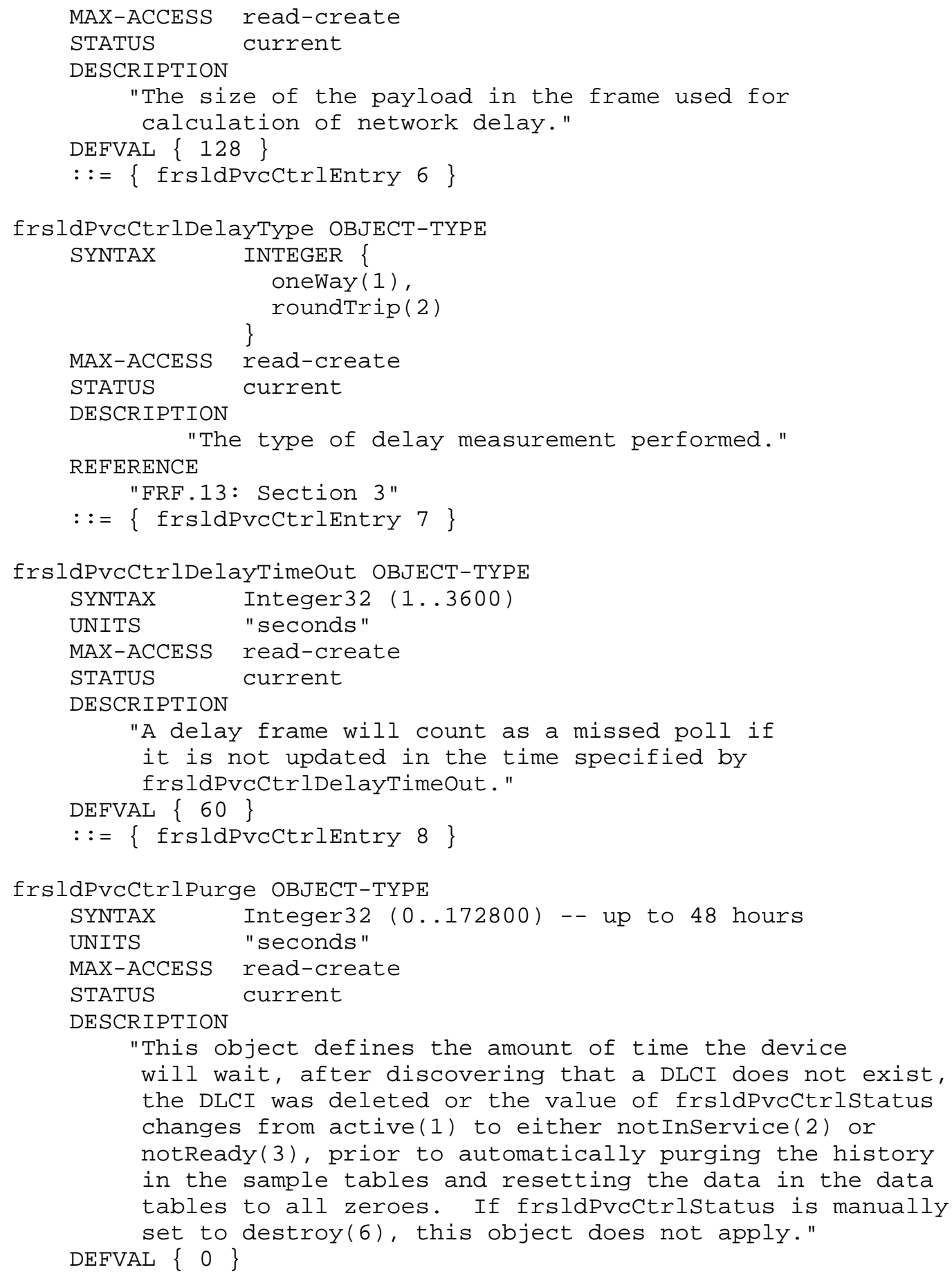




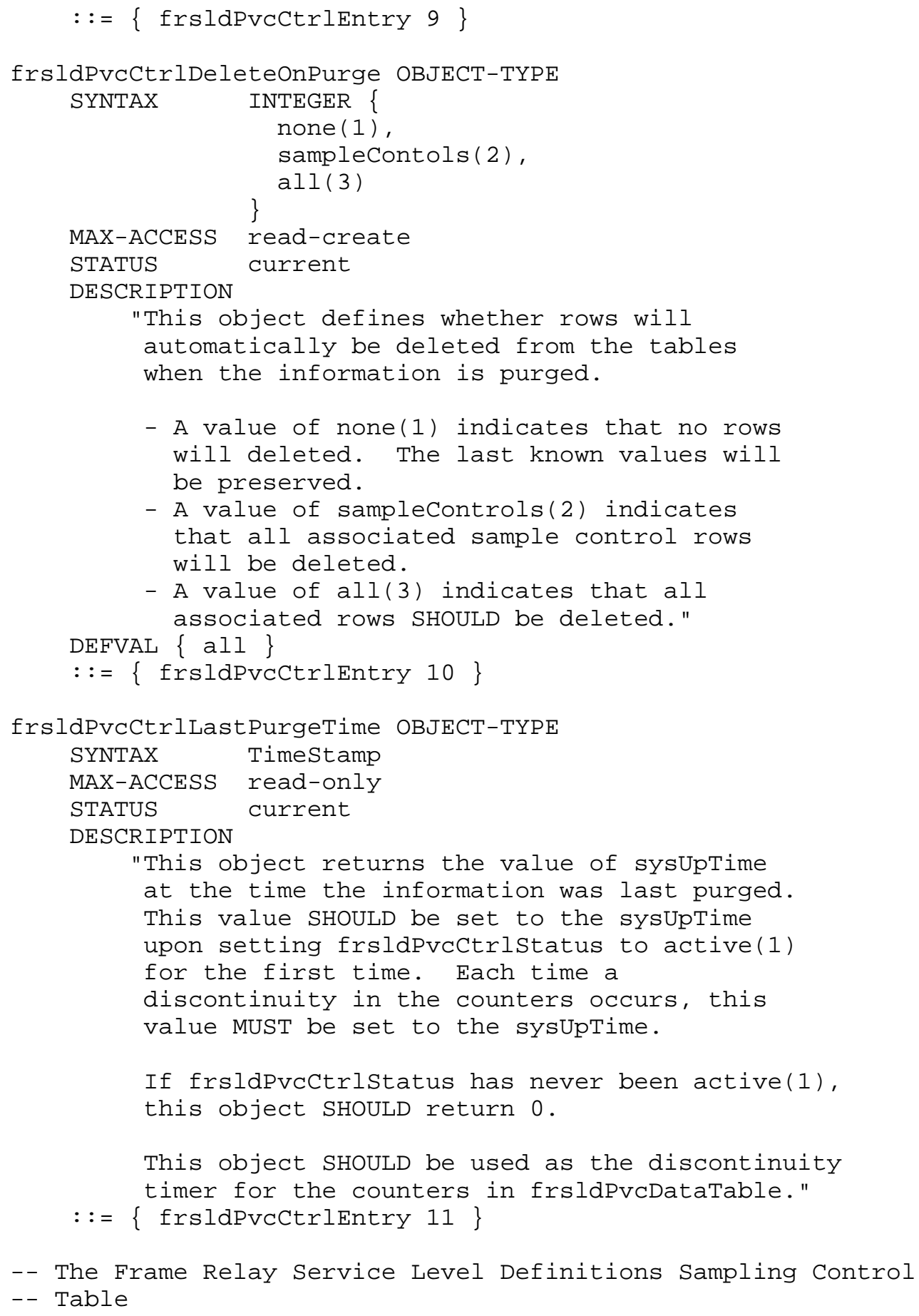




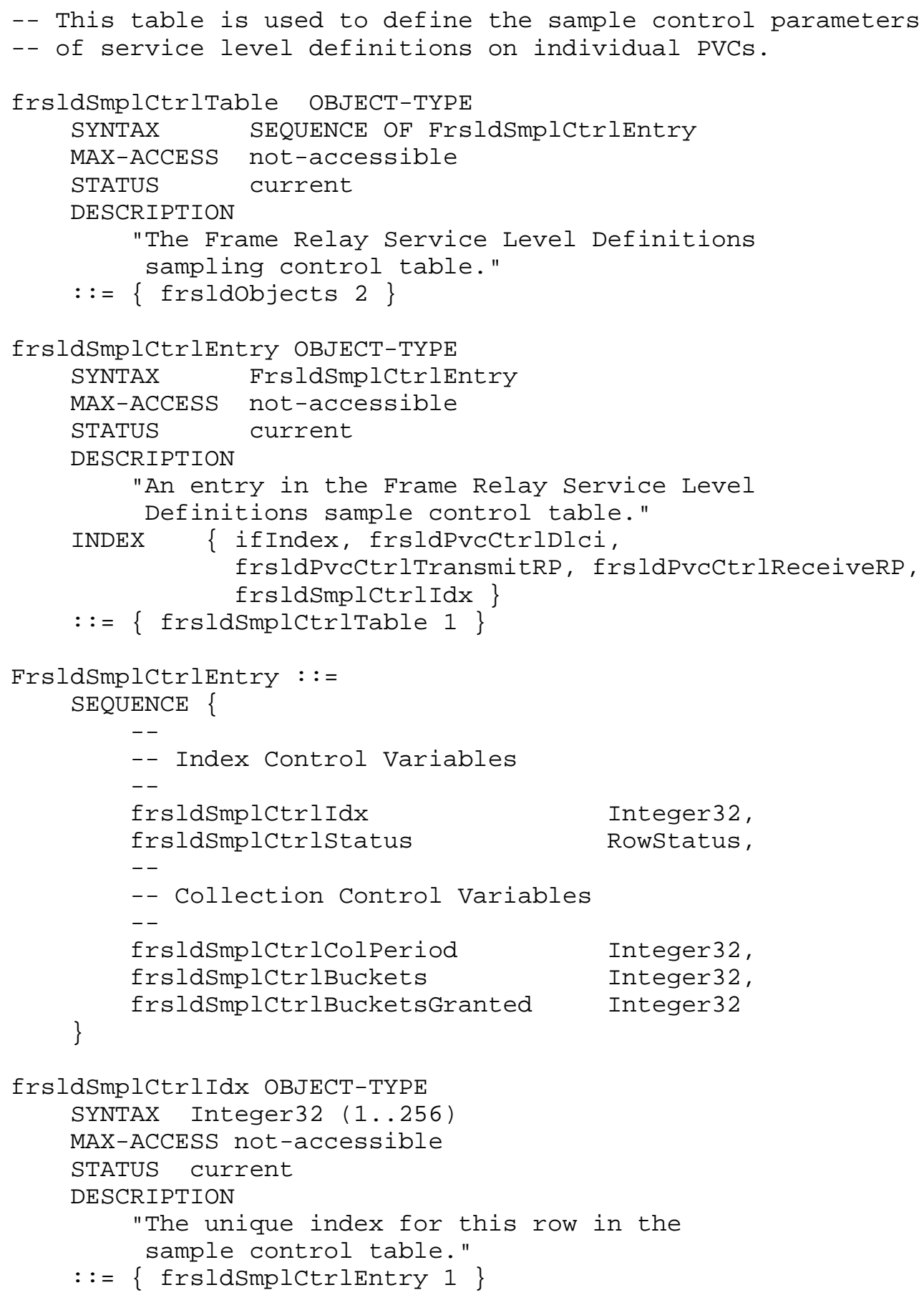




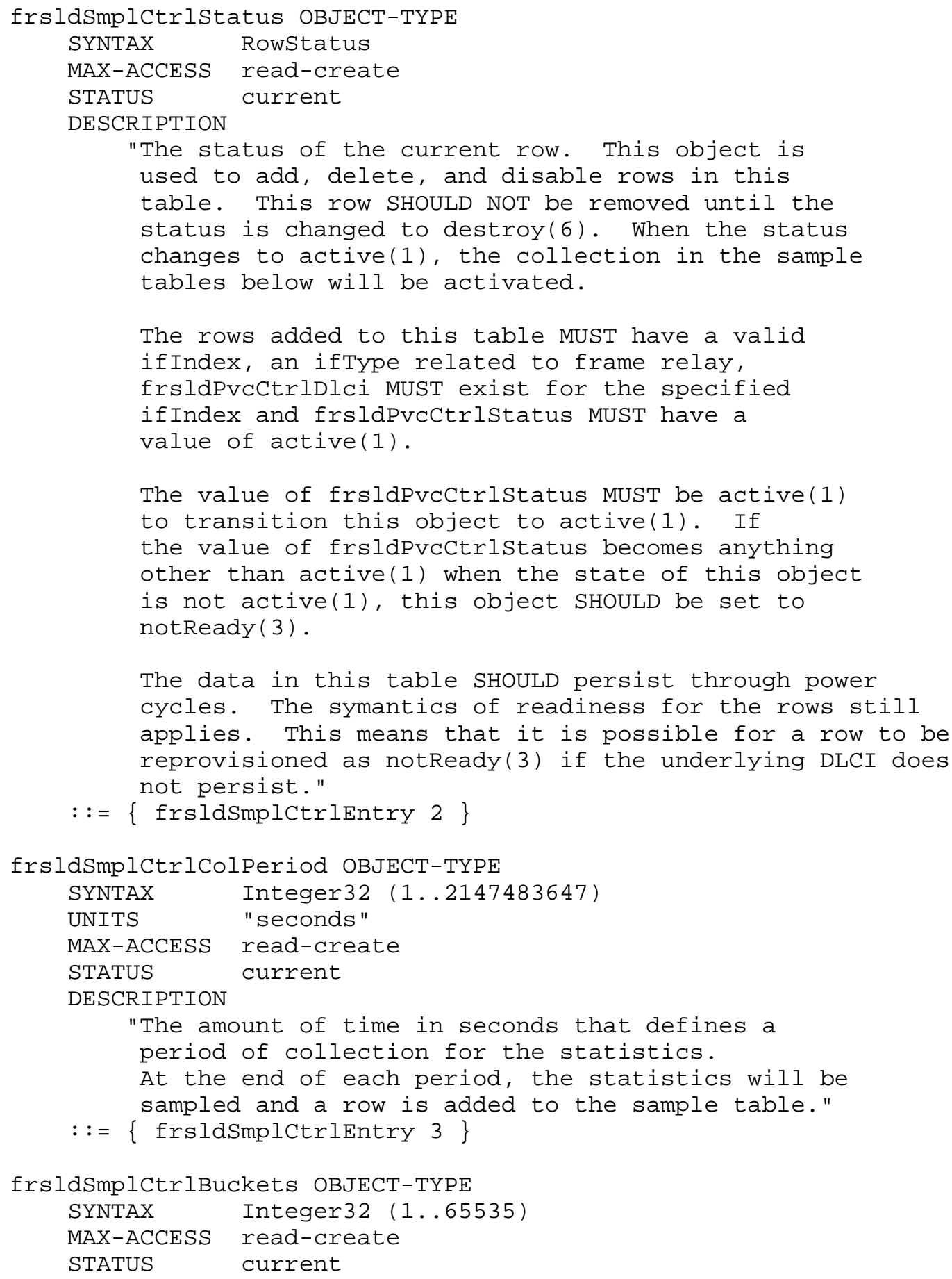


DESCRIPTION

"The number of discrete buckets over which the data statistics are sampled.

When this object is created or modified, the device SHOULD attempt to set the frsldSmplCtrlBucketsGranted to a value as close as is possible depending upon the implementation and the available resources."

DEFVAL $\{60\}$

$::=\{$ frsldSmplCtrlentry 4$\}$

frsldSmplCtrlBucketsGranted OBJECT-TYPE

SYNTAX Integer32 (0.65535)

MAX-ACCESS read-only

STATUS current

DESCRIPTION

"The number of discrete buckets granted. This object will return 0 until frsldSmplctrlstatus is set to active(1). At that time the buckets will be allocated depending upon implementation and available resources."

$::=\{$ frsldSmplCtrlentry 5$\}$

-- The Frame Relay Service Level Definitions PVC Data Table

$--$

-- This table contains the accumulated values of

-- the collected data. This table is the table that should

-- be referenced by external polling mechanisms if time

-- based polling be desired.

frsldPvcDataTable OBJECT-TYPE

SYNTAX SEQUENCE OF FrsldPvCDataEntry

MAX-ACCESS not-accessible

STATUS current

DESCRIPTION

"The Frame Relay Service Level Definitions

data table.

This table contains accumulated values of the collected data. It is the table that should be referenced by external polling mechanisms if time based polling be desired." $::=\{$ frsldobjects 3$\}$

frsldPvcDataEntry OBJECT-TYPE

SYNTAX FrsldPvCDataEntry

MAX-ACCESS not-accessible 


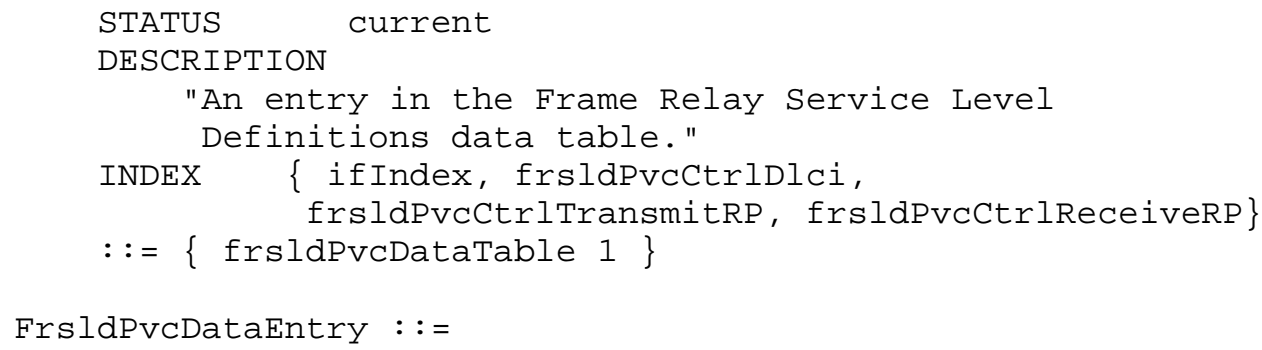

frsldPvCDataMissedPolls OBJECT-TYPE SYNTAX Counter32

MAX-ACCESS read-only

STATUS current

DESCRIPTION

"The total number of polls that have been determined to be missed. These polls are typically associated with the calculation of delay but may also be used for the calculation of other statistics. If an anticipated poll is not received in a reasonable amount of time, it should be counted as missed. The value used to determine the reasonable amount of time is contained in frsldPvcCtrlDelayTimeout.

Discontinuities in the value of this counter can occur at re-initialization of the management system and at other times as indicated by 
$:: \begin{aligned} & \text { frsldPvcCtrllastPurgeTime. " } \\ & :=\{\text { frsldPveDataEntry } 1\}\end{aligned}$

frsldPvCDataFrDeliveredC OBJECT-TYPE

SYNTAX Counter32

MAX-ACCESS read-only

STATUS current

DESCRIPTION

"The number of frames that were received at frsldPvcCtrlReceiveRP and determined to have been sent within CIR.

Discontinuities in the value of this counter can occur at re-initialization of the management system and at other times as indicated by frsldPvcCtrllastPurgeTime."

REFERENCE

"FRF.13: Section 4.1 (FramesDeliveredc) "

$::=\{$ frsldPveDataEntry 2$\}$

frsldPvcDataFrDeliveredE OBJECT-TYPE

SYNTAX Counter32

MAX-ACCESS read-only

STATUS current

DESCRIPTION

"The number of frames that were received at

frsldPvcCtrlReceiveRP and determined to have been

sent in excess of the CIR.

Discontinuities in the value of this counter can

occur at re-initialization of the management system and at other times as indicated by frsldPvcCtrlLastPurgeTime."

REFERENCE

"FRF.13: Section 4.1 (FramesDeliverede)"

$::=\{$ frsldPvcDataEntry 3$\}$

frsldPvcDataFrofferedC OBJECT-TYPE

SYNTAX Counter32

MAX-ACCESS read-only

STATUS current

DESCRIPTION

"The number of frames that were offered through

frsldPvcCtrlTransmitRP within CIR.

Discontinuities in the value of this counter can occur at re-initialization of the management system and at other times as indicated by 


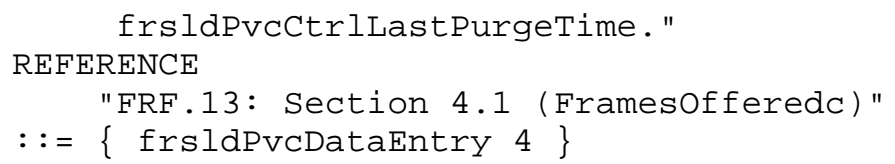




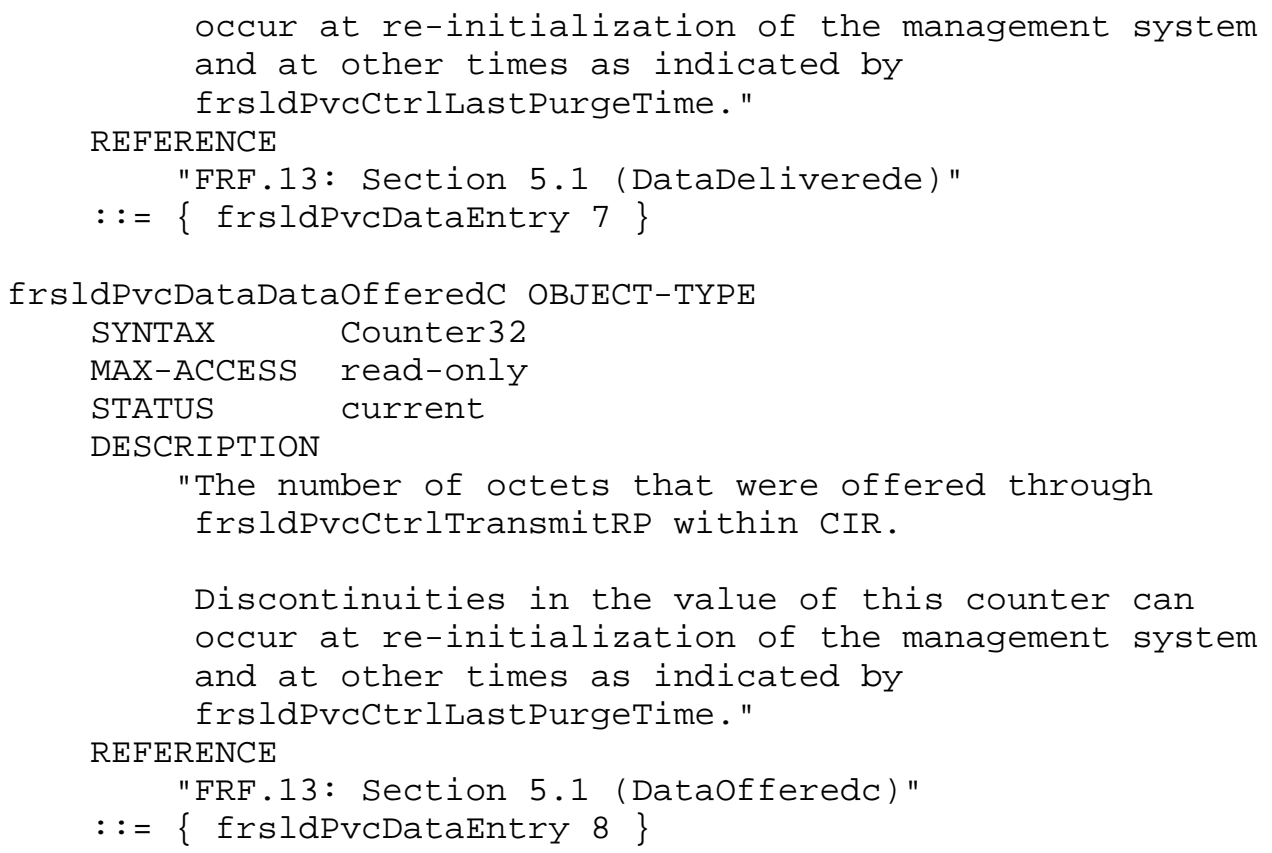




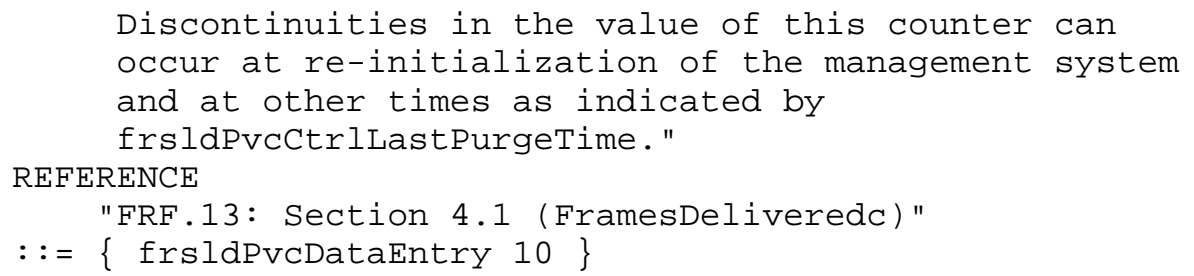




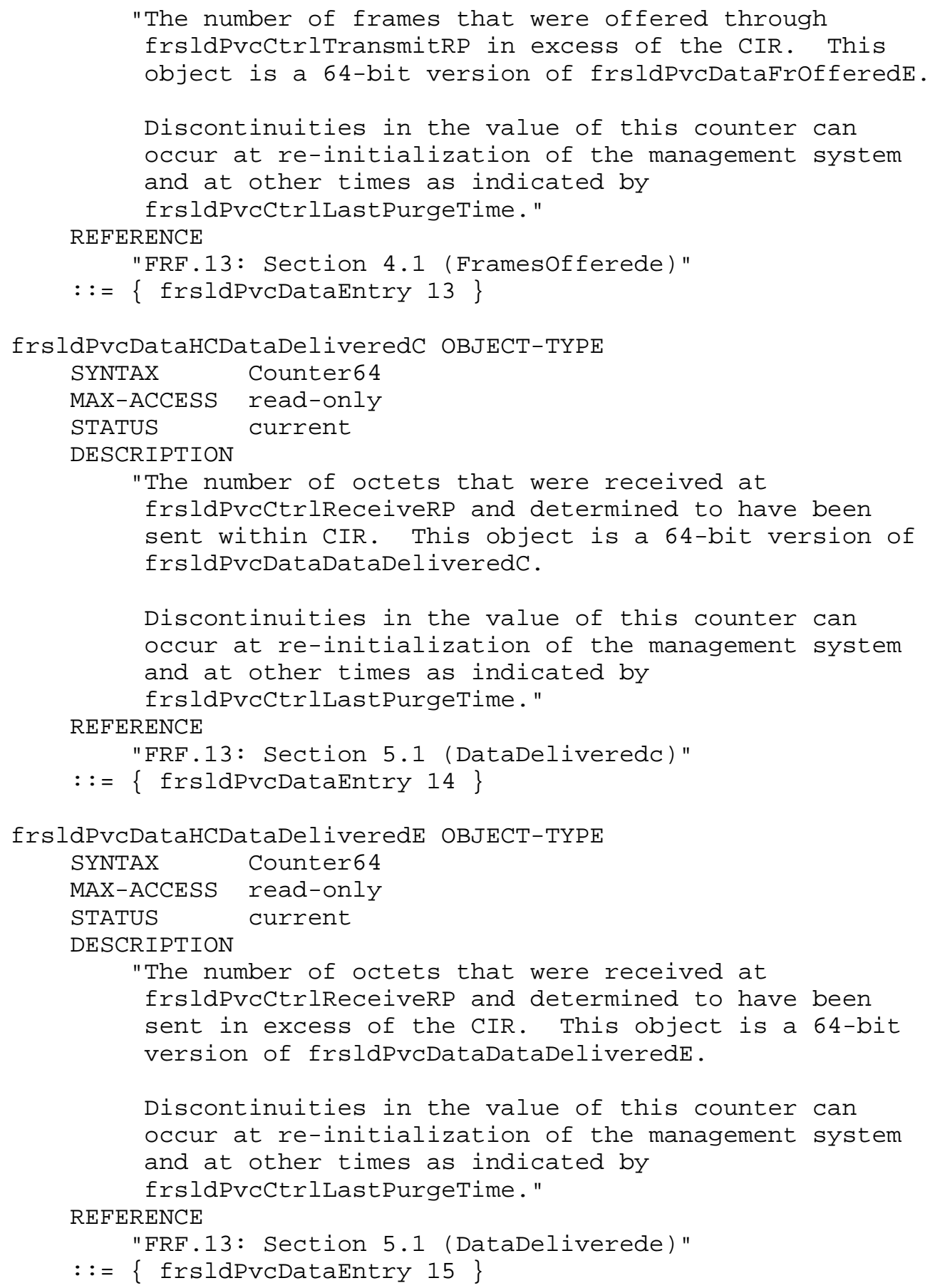




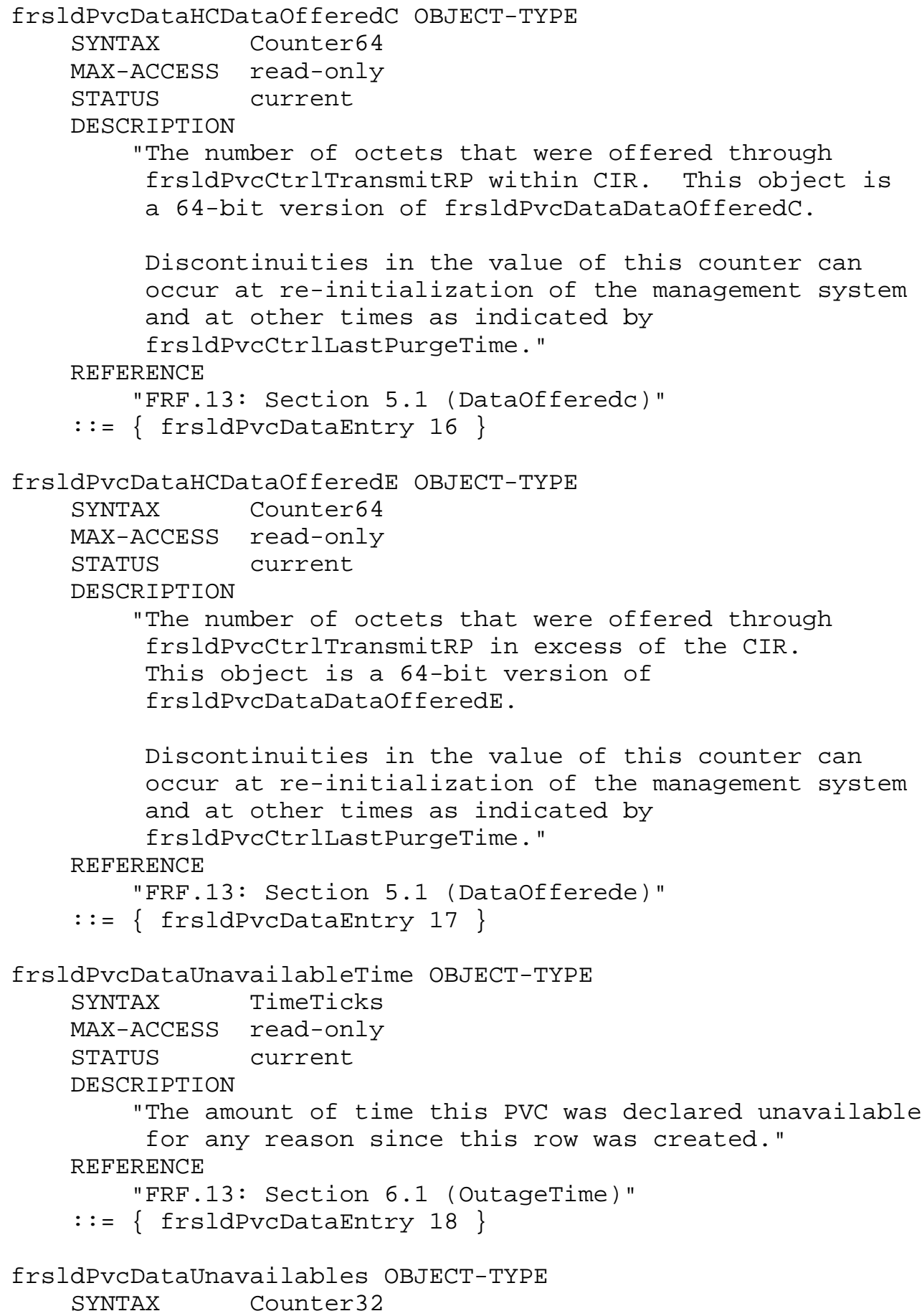




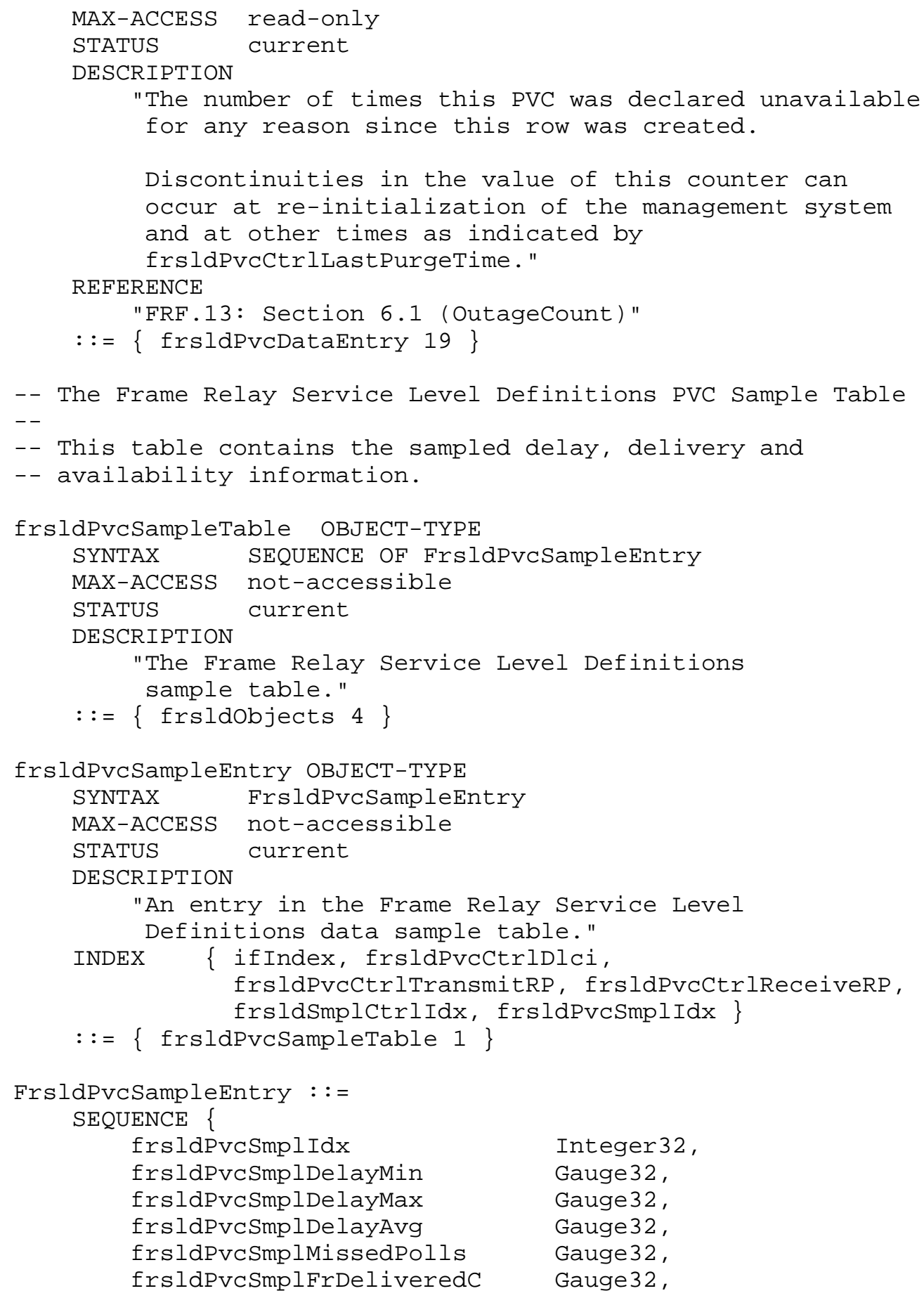




frsldPvcSmplFrDeliveredE
frsldPvcSmplFrOfferedC
frsldPvcSmplFrOfferedE
frsldPvcSmplDataDeliveredC
frsldPvcSmplDataDeliveredE
frsldPvcSmplDataOfferedC
frsldPvcSmplDataOfferedE
frsldPvcSmplHCFrDeliveredC
frsldPvcSmplHCFrDeliveredE
frsldPvcSmplHCFrOfferedC
frsldPvcSmplHCFrOfferedE
frsldPvcSmplHCDataDeliveredC
frsldPvcSmplHCDataDeliveredE
frsldPvcSmplHCDataOfferedC
frsldPvcSmplHCDataOfferedE
frsldPvcSmplUnavailableTime
frsldPvcSmplUnavailables
frsldPvcSmplStartTime
frsldPvcSmplEndTime

frsldPvcSmplFrDeliveredE

vicsmplfrofferedC

frsldPvcSmplDataDeliveredC

frsldPvcSmplDataOfferedC

frsldPvcSmplDataOfferedE

frsldPvCSmpl HCF rDeliveredE

frsldPvcSmpl HCFrOfferedC

frsldPvCSmpl HCFrofferedE

frsldPvcSmpl HCDataDeliveredE

frsldPvcSmplHCDataOfferedC

frsldPvcSmpl HCDataOf feredE

frsldPvcSmplUnavailableTime

frsldPvcSmplUnavailables

frsldPvcSmplEndTime

)

frsldPvCSmplIdx OBJECT-TYPE

SYNTAX Integer32 (1..2147483647)

MAX-ACCESS not-accessible

STATUS

current

DESCRIPTION

"The bucket index of the current sample. This increments once for each new bucket in the table."

$::=\{$ frsldPvcSampleEntry 1$\}$

frsldPvcSmplDelayMin OBJECT-TYPE

SYNTAX Gauge32

UNITS "microseconds"

MAX-ACCESS read-only

STATUS current

DESCRIPTION

"The minimum delay reported in microseconds measured for any information packet that arrived during this interval.

A value of zero means that no data is available." REFERENCE

"FRF.13: Section 3.1 (FTD) "

$::=\{$ frsldPvcSampleEntry 2$\}$

frsldPvCSmplDelayMax OBJECT-TYPE

SYNTAX Gauge32 


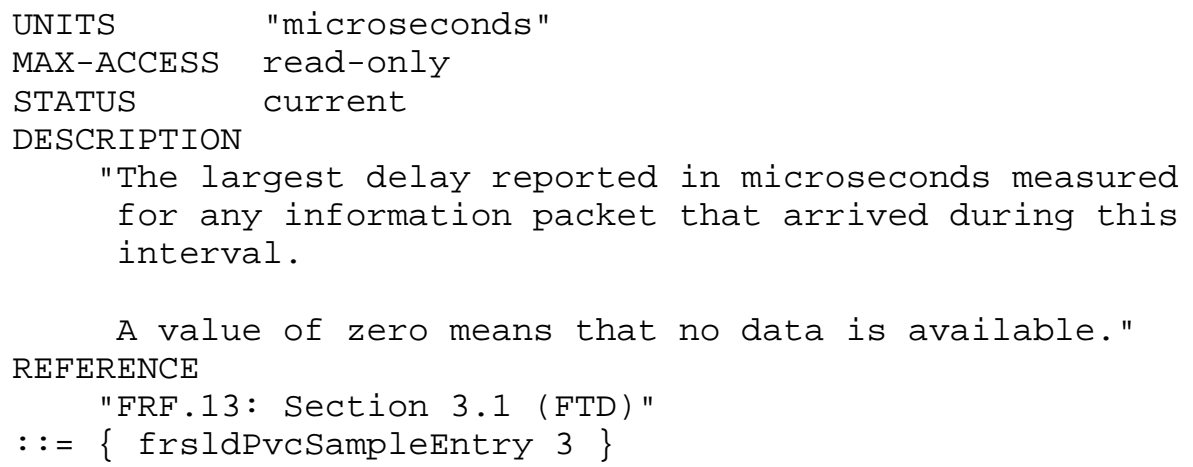




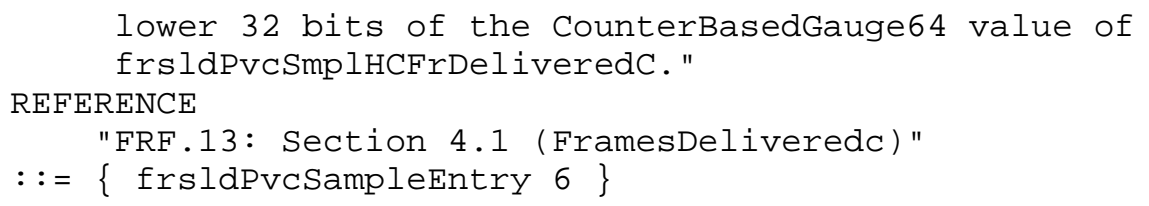




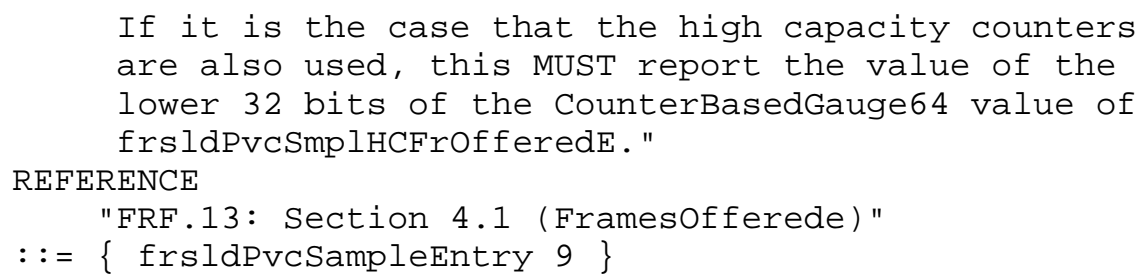

"The number of octets that were offered through 


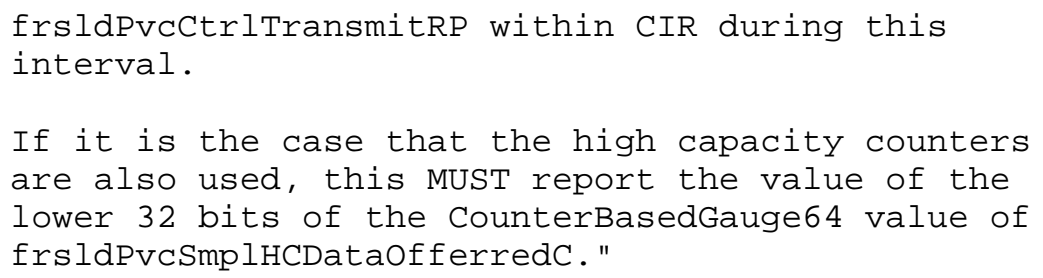




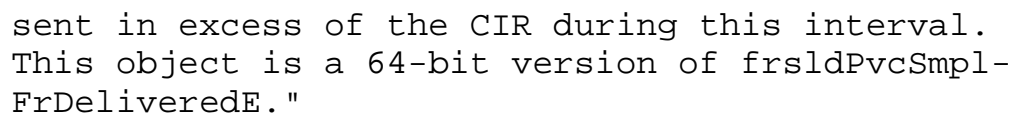




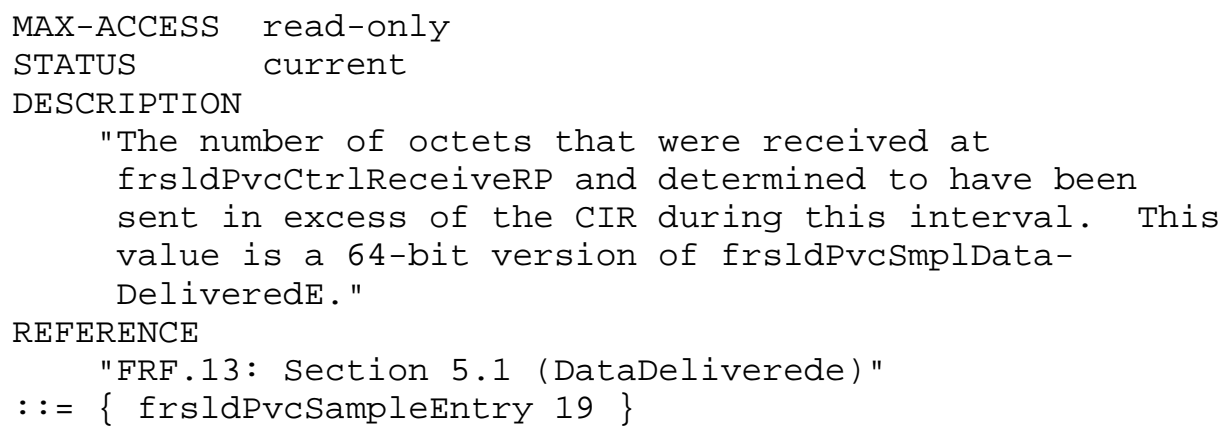




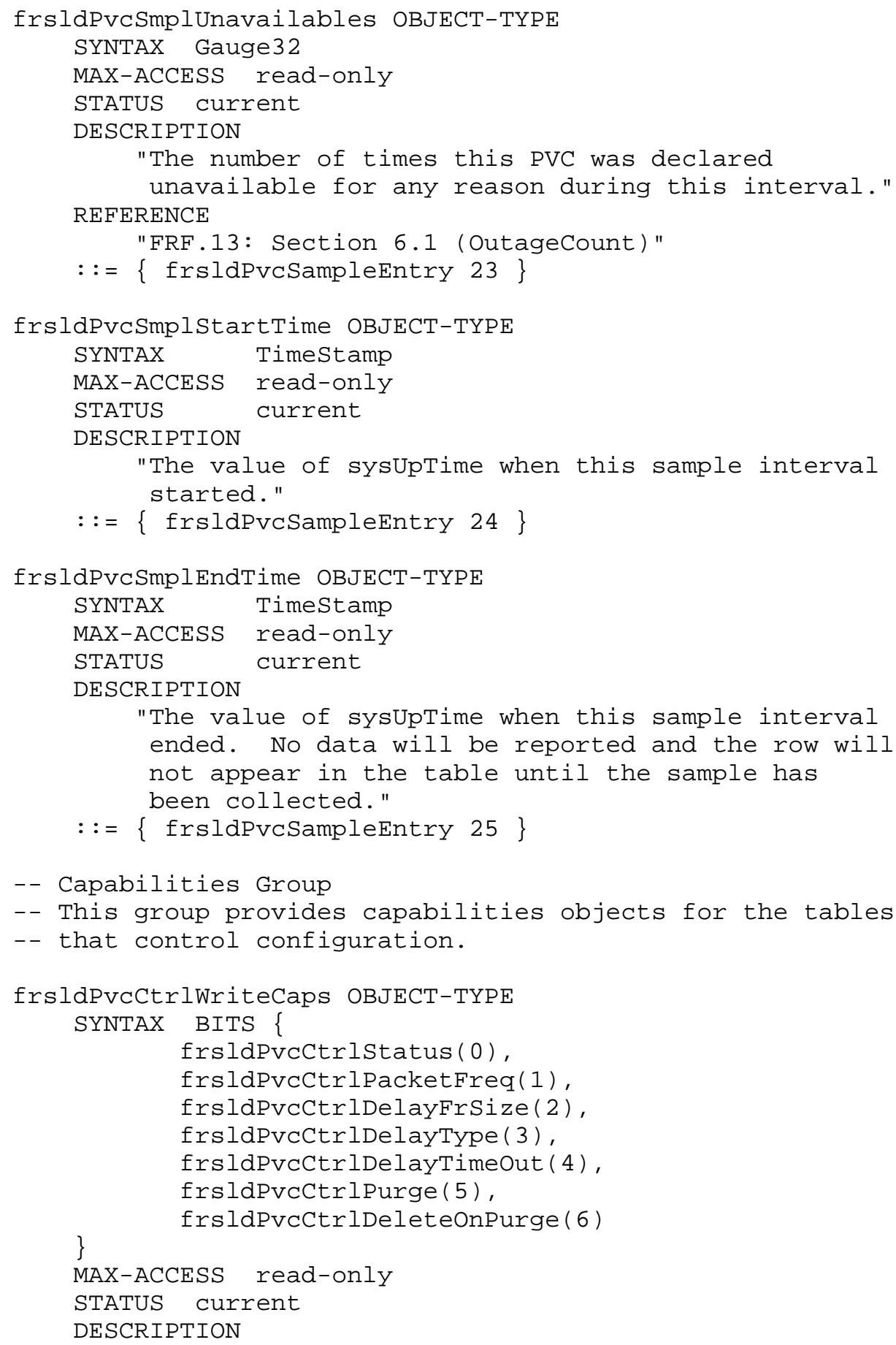




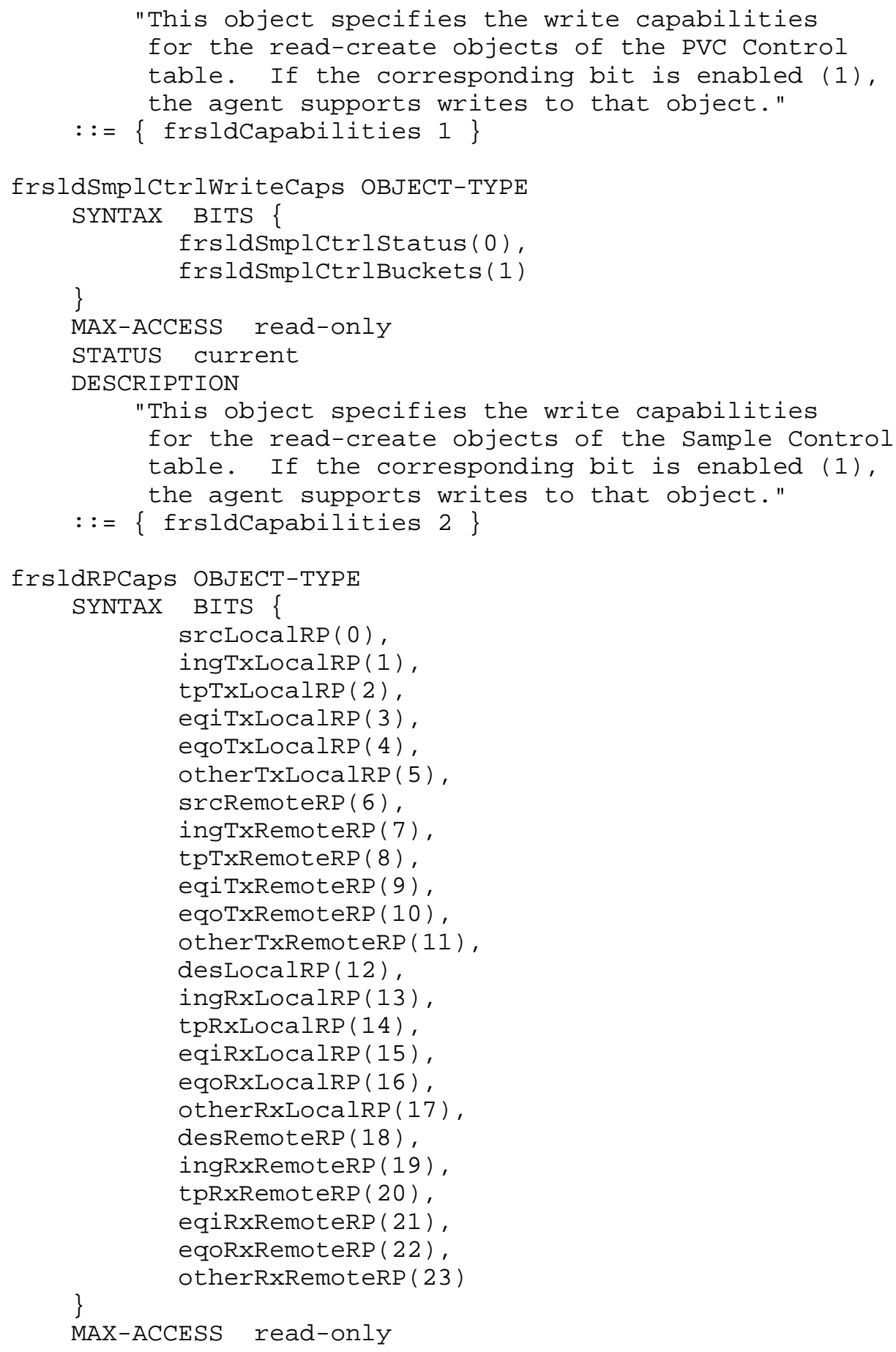




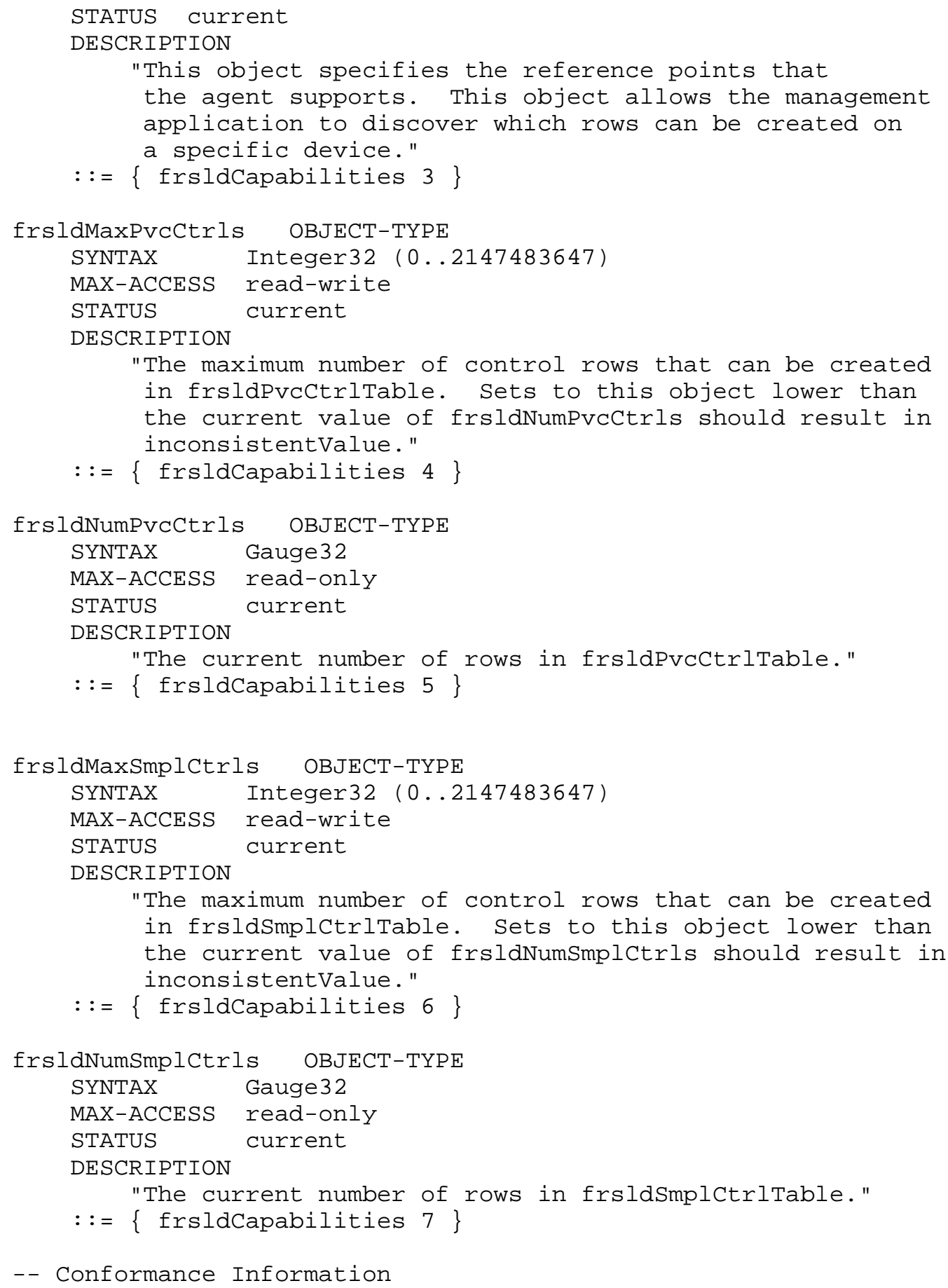




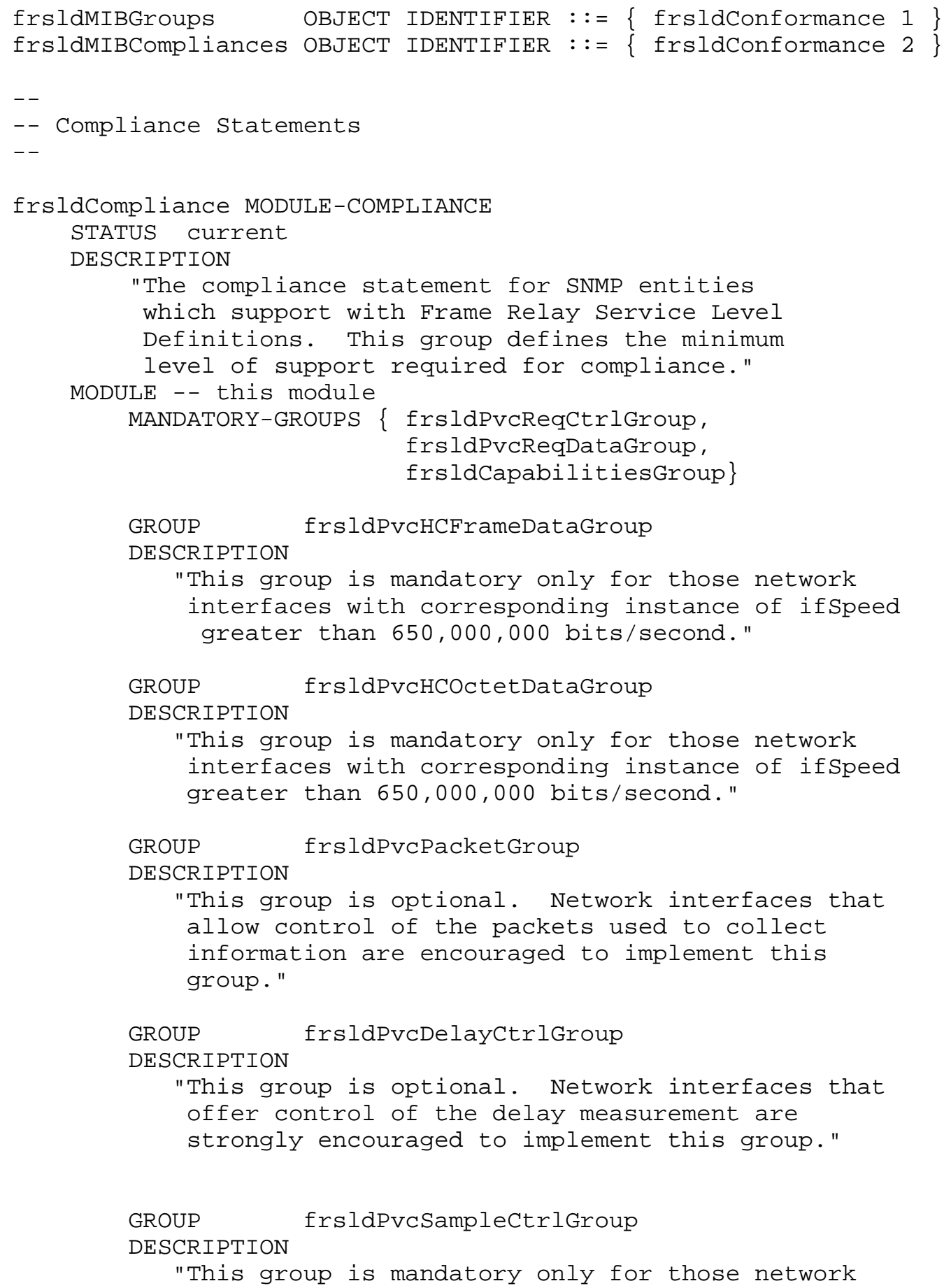




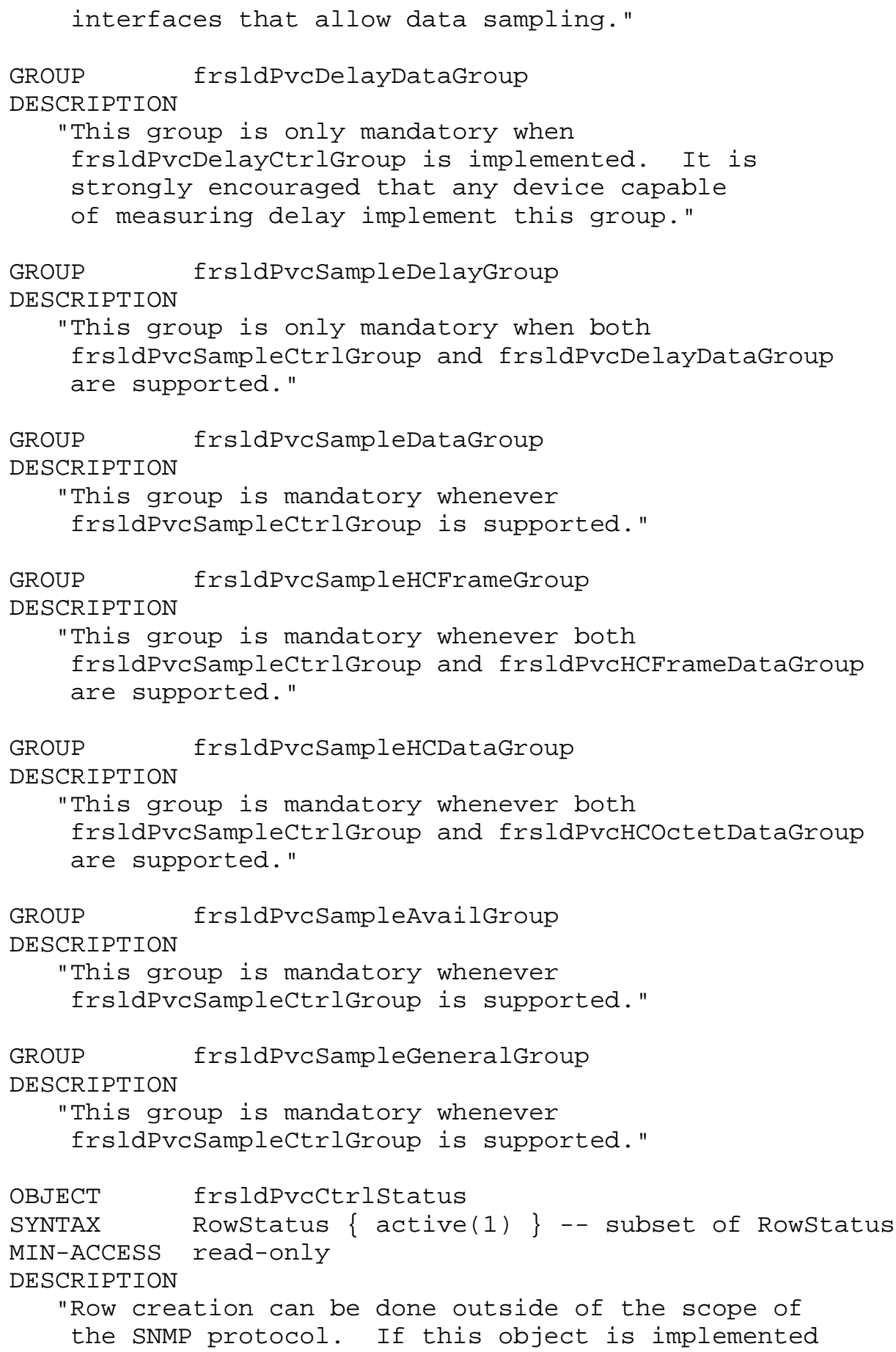


-- Units of Conformance

$--$

frsldPvCReqCtrlGroup OBJECT-GROUP

OBJECTS \{

frsldPvcCtrlstatus,

frsldPvcCtrlPurge,

frsldPvcCtrlDeleteonPurge,

\}

frsldPvcCtrlLastPurgeTime

STATUS current

DESCRIPTION

"A collection of required objects providing control information applicable to a PVC which implements Service Level Definitions."

$::=\{$ frsldMIBGroups 1$\}$

frsldPvcPacketGroup OBJECT-GROUP

OBJECTS \{

frsldPvcCtrlPacketFreq

\}

STATUS current

DESCRIPTION

"A collection of optional objects providing packet level control information applicable to a PVC which implements Service Level Definitions."

$::=\{$ frsldMIBGroups 2$\}$

frsldPvcDelayCtrlGroup OBJECT-GROUP

OBJECTS \{

frsldPvcCtrlDelayFrSize,

frsldPvcCtrlDelaytype,

\}

frsldPvcCtrlDelaytimeout

STATUS current

DESCRIPTION

"A collection of optional objects providing delay control information applicable to a PVC which implements Service Level Definitions.

If this group is implemented, frsldPvcPacketGroup and frsldPvcDelayDataGroup MUST also be implemented." $::=\{$ frsldMIBGroups 3$\}$

frsldPvcSampleCtrlGroup OBJECT-GROUP

OBJECTS \{

frsldSmplCtrlstatus, frsldSmplCtrlColperiod, frsldSmplCtrlBuckets, 


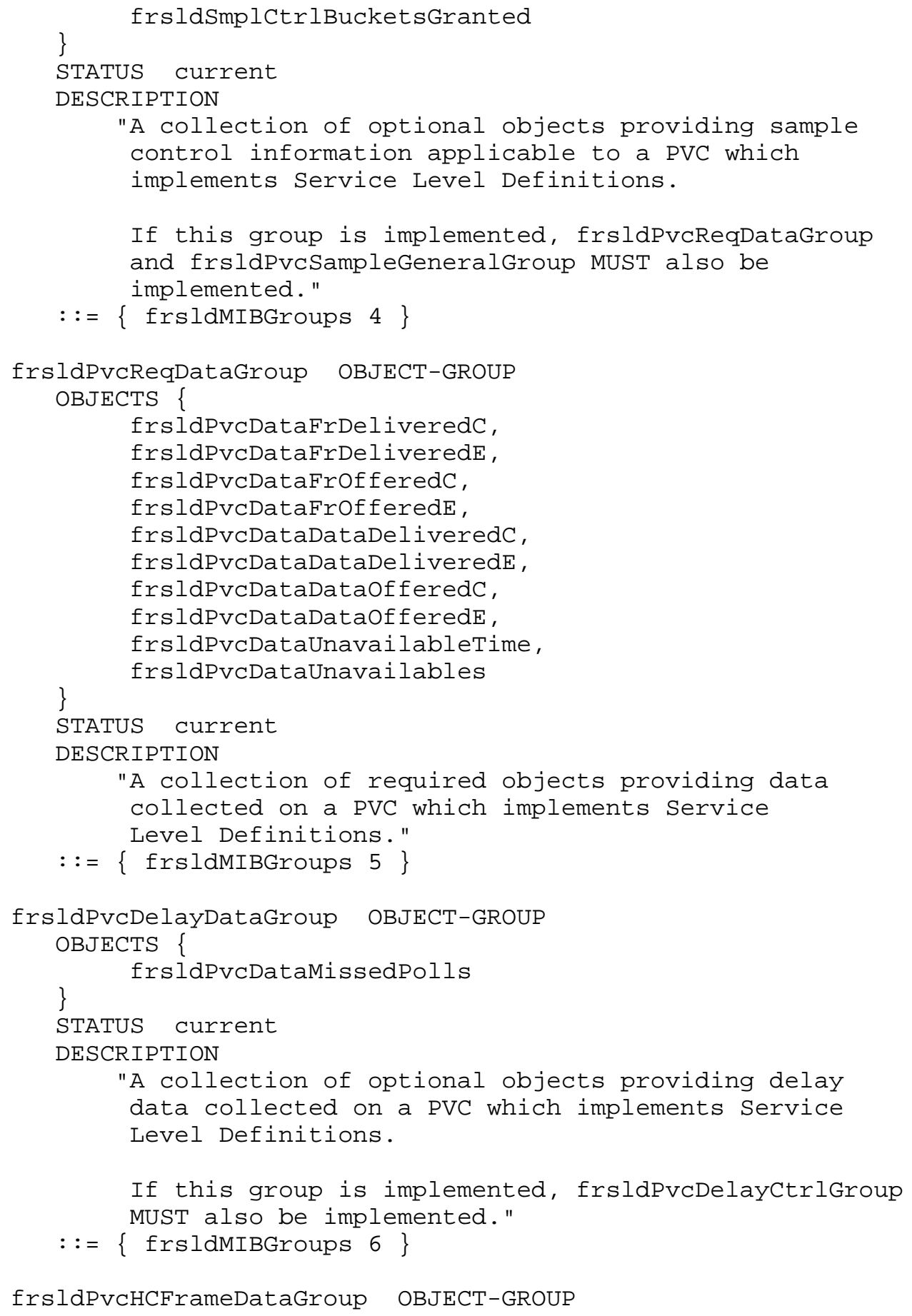




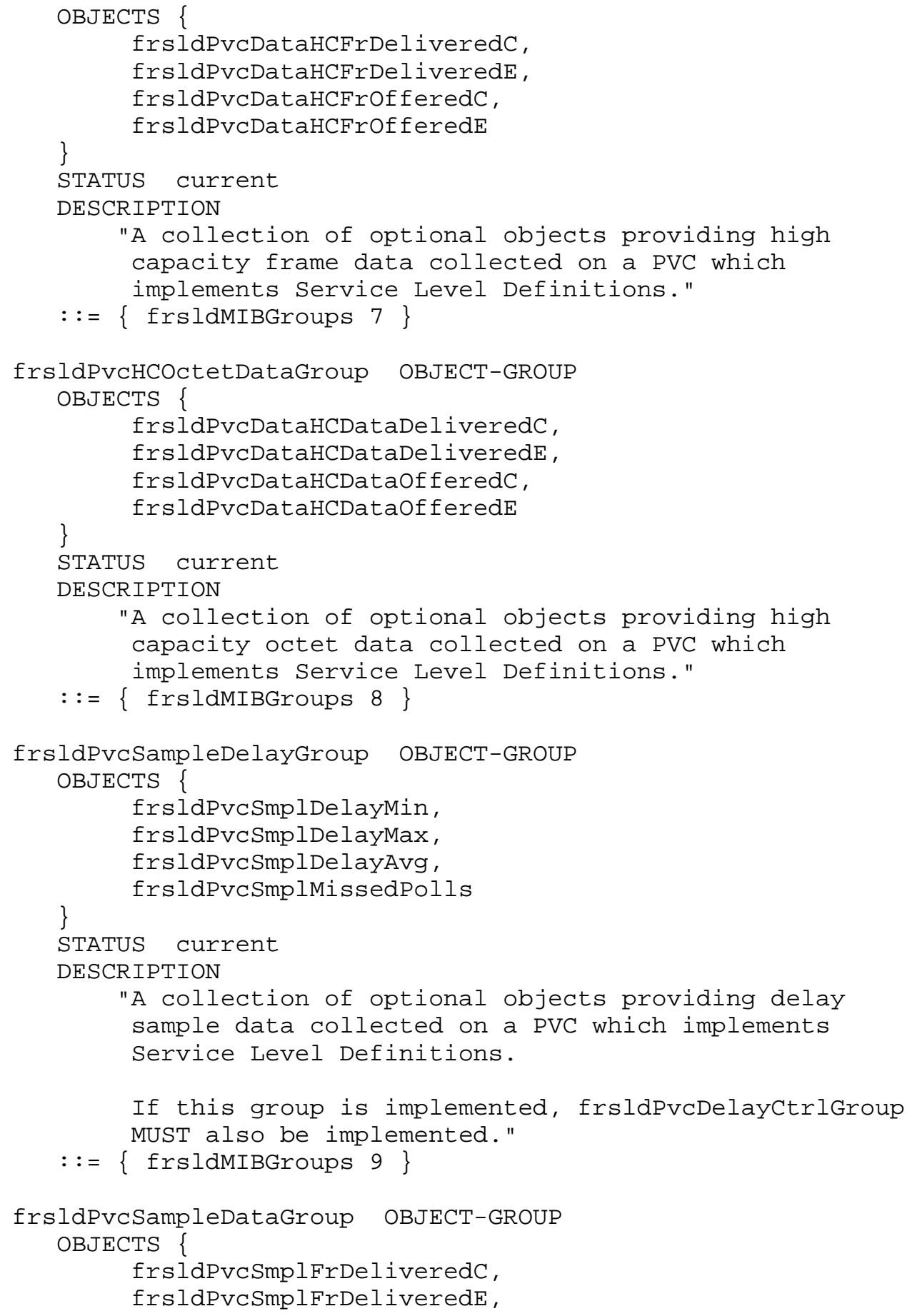




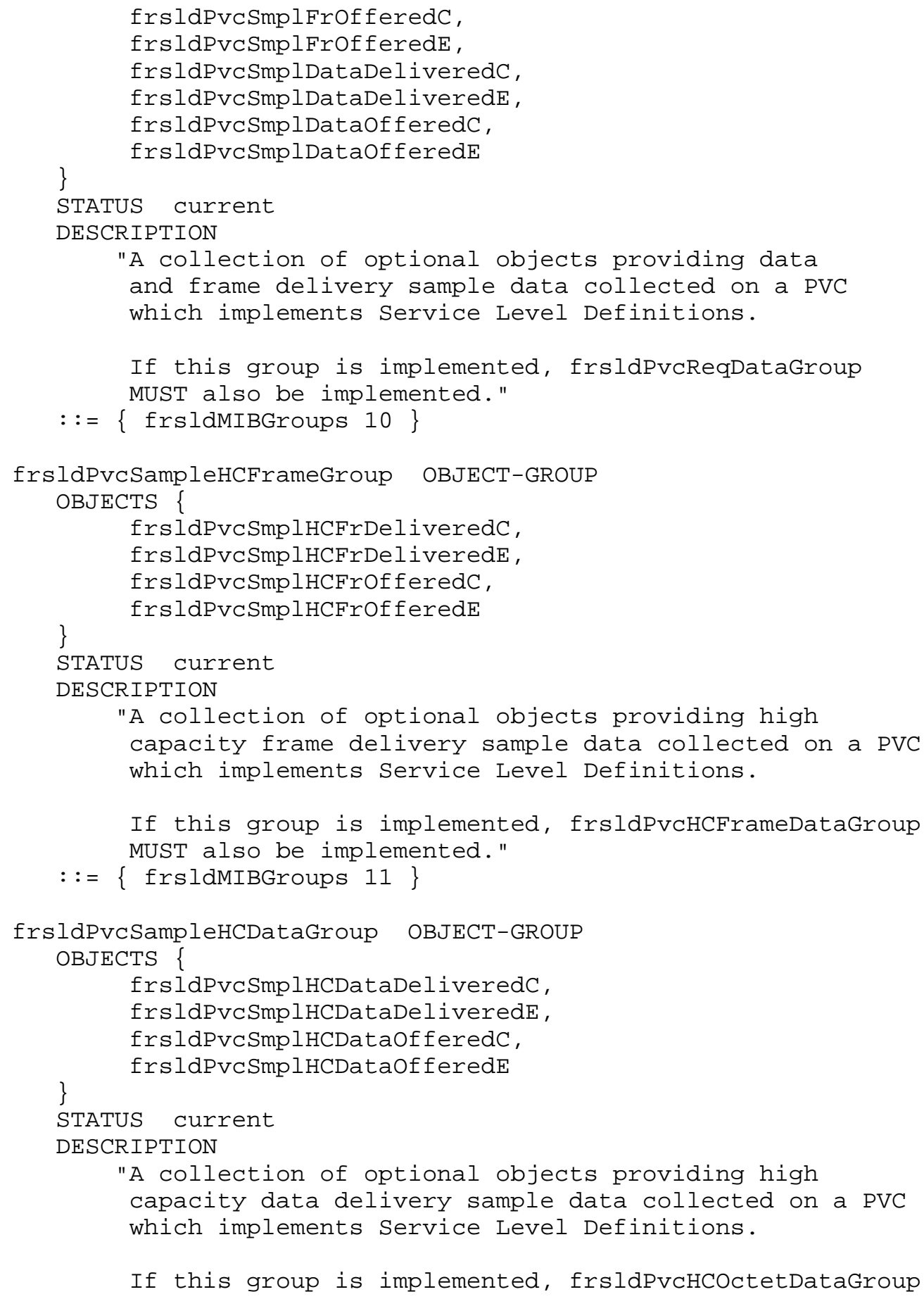




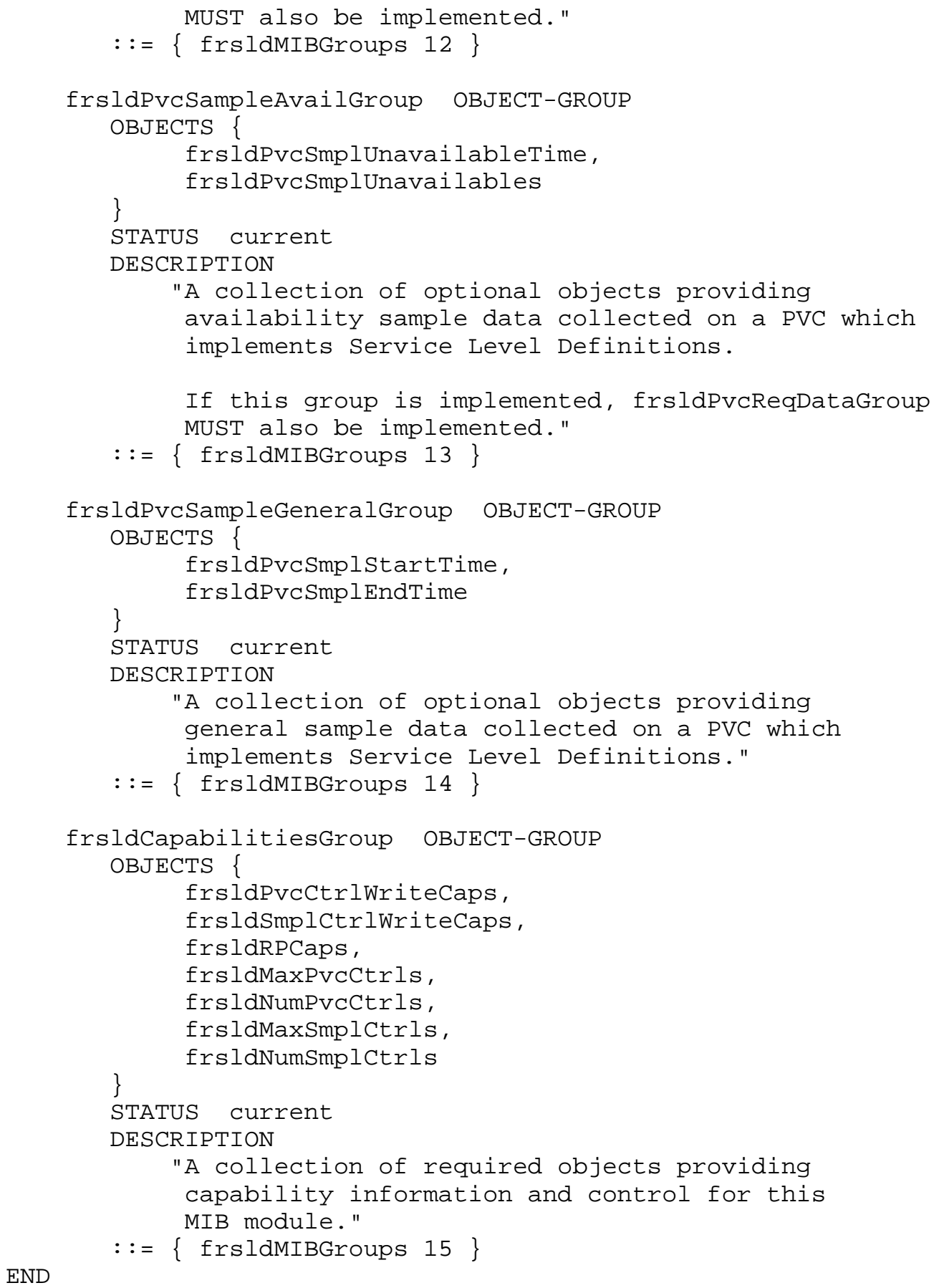

END

Steinberger \& Nicklass Standards Track [Page 60] 


\section{Acknowledgments}

This document was produced by the Frame Relay Service MIB Working Group. It is based on the Frame Relay Forum's implementation agreement on service level definitions, FRF.13 [17].

The editors would like to thank the following people for their helpful comments:

- Ken Rehbehn, Visual Networks

- Santa Dasu, Quick Eagle Networks

9. References

[1] Harrington, D., Presuhn, R. and B. Wijnen, "An Architecture for Describing SNMP Management Frameworks", RFC 2571, April 1999.

[2] Rose, M. and K. McCloghrie, "Structure and Identification of Management Information for TCP/IP-based Internets", STD 16, RFC 1155, May 1990.

[3] Rose, M. and K. MCCloghrie, "Concise MIB Definitions", STD 16, RFC 1212, March 1991.

[4] Rose, M., "A Convention for Defining Traps for use with the SNMP", RFC 1215, March 1991.

[5] McCloghrie, K., Perkins, D., Schoenwaelder, J., Case, J., Rose, M. and S. Waldbusser, "Structure of Management Information Version 2 (SMIv2)", STD 58, RFC 2578, April 1999.

[6] McCloghrie, K., Perkins, D., Schoenwaelder, J., Case, J., Rose, M. and S. Waldbusser, "Textual Conventions for SMIV2", STD 58, RFC 2579, April 1999.

[7] McCloghrie, K., Perkins, D., Schoenwaelder, J., Case, J., Rose, M. and S. Waldbusser, "Conformance Statements for SMIv2", STD 58, RFC 2580, April 1999.

[8] Case, J., Fedor, M., Schoffstall, M. and J. Davin, "Simple Network Management Protocol", STD 15, RFC 1157, May 1990. 
[9] Case, J., McCloghrie, K., Rose, M. and S. Waldbusser, "Introduction to Community-based SNMPv2", RFC 1901, January 1996.

[10] Case, J., McCloghrie, K., Rose, M. and S. Waldbusser, "Transport Mappings for Version 2 of the Simple Network Management Protocol (SNMPv2) ", RFC 1906, January 1996.

[11] Case, J., Harrington D., Presuhn R. and B. Wijnen, "Message Processing and Dispatching for the Simple Network Management Protocol (SNMP)", RFC 2572, April 1999.

[12] Blumenthal, U. and B. Wijnen, "User-based Security Model (USM) for version 3 of the Simple Network Management Protocol (SNMPv3)", RFC 2574, April 1999.

[13] Case, J., McCloghrie, K., Rose, M. and S. Waldbusser, "Protocol Operations for Version 2 of the Simple Network Management Protocol (SNMPV2)", RFC 1905, January 1996.

[14] Levi, D., Meyer, P. and B. Stewart, "SNMPv3 Applications", RFC 2573, April 1999.

[15] Wijnen, B., Presuhn, R. and K. McCloghrie, "View-based Access Control Model (VACM) for the Simple Network Management Protocol (SNMP) ", RFC 2575, April 1999.

[16] Case, J., Mundy, R., Partain, D. and B. Stewart, "Introduction to Version 3 of the Internet-standard Network Management Framework", RFC 2570, April 1999.

[17] Frame Relay Forum Technical Committee, "Service Level Definitions Implementations Agreement", FRF.13, August 1998.

[18] Rehbehn, K. and D. Fowler, "Definitions of Managed Objects for Frame Relay Service", RFC 2954, October 2000.

[19] Waldbusser, S., "Remote Network Monitoring Management Information Base Version 2 using SMIv2", RFC 2021, January 1997.

[20] Brown, C. and F. Baker, "Management Information Base for Frame Relay DTEs Using SMIv2", RFC 2115, September 1997.

[21] McCloghrie, K. and F. Kastenholz, "The Interfaces Group MIB", RFC 2863, June 2000 .

[22] Bradner, S., "Key words for use in RFCs to Indicate Requirement Levels", BCP 14, RFC 2119, March 1997. 


\section{Security Considerations}

There are a number of management objects defined in this MIB that have a MAX-ACCESS clause of read-write and/or read-create. Such objects may be considered sensitive or vulnerable in some network environments. The support for SET operations in a non-secure environment without proper protection can have a negative effect on network operations.

SNMPv1 by itself is not a secure environment. Even if the network itself is secure (for example by using IPSec), even then, there is no control as to who on the secure network is allowed to access and GET/SET (read/change/create/delete) the objects in this MIB.

It is recommended that the implementers consider the security features as provided by the SNMPv3 framework. Specifically, the use of the User-based Security Model RFC 2574 [12] and the View-based Access Control Model RFC 2575 [15] is recommended.

It is then a customer/user responsibility to ensure that the SNMP entity giving access to an instance of this MIB, is properly configured to give access to the objects only to those principals (users) that have legitimate rights to indeed GET or SET (change/create/delete) them.

\section{Authors' Addresses}

Robert Steinberger

Fujitsu Network Communications

2801 Telecom Parkway

Richardson, TX 75082

Phone: 1-972-479-4739

EMail: robert.steinbergerefnc.fujitsu.com

Orly Nicklass, Ph.D

RAD Data Communications Ltd.

12 Hanechoshet Street

Tel Aviv, Israel 69710

Phone: 97237659969

EMail: Orly_n@rad.co.il 


\section{Full Copyright statement}

Copyright (C) The Internet Society (2002). All Rights Reserved.

This document and translations of it may be copied and furnished to others, and derivative works that comment on or otherwise explain it or assist in its implementation may be prepared, copied, published and distributed, in whole or in part, without restriction of any kind, provided that the above copyright notice and this paragraph are included on all such copies and derivative works. However, this document itself may not be modified in any way, such as by removing the copyright notice or references to the Internet society or other Internet organizations, except as needed for the purpose of developing Internet standards in which case the procedures for copyrights defined in the Internet Standards process must be followed, or as required to translate it into languages other than English.

The limited permissions granted above are perpetual and will not be revoked by the Internet society or its successors or assigns.

This document and the information contained herein is provided on an "AS IS" basis and THE INTERNET SOCIETY AND THE INTERNET ENGINEERING TASK FORCE DISCLAIMS ALL WARRANTIES, EXPRESS OR IMPLIED, INCLUDING BUT NOT LIMITED TO ANY WARRANTY THAT THE USE OF THE INFORMATION HEREIN WILL NOT INFRINGE ANY RIGHTS OR ANY IMPLIED WARRANTIES OF MERCHANTABILITY OR FITNESS FOR A PARTICULAR PURPOSE.

Acknowledgement

Funding for the RFC Editor function is currently provided by the Internet society. 